\title{
New Energy Analysis of Yee Scheme for Metamaterial Maxwell's Equations on Non-Uniform Rectangular Meshes
}

\author{
Xixian Bai and Hongxing Rui* \\ School of Mathematics, Shandong University, Jinan 250100, Shandong, China
}

Received 8 July 2020; Accepted (in revised version) 23 September 2020

\begin{abstract}
In this paper, several new energy identities of metamaterial Maxwell's equations with the perfectly electric conducting (PEC) boundary condition are proposed and proved. These new energy identities are different from the Poynting theorem. By using these new energy identities, it is proved that the Yee scheme on non-uniform rectangular meshes is stable in the discrete $L^{2}$ and $H^{1}$ norms when the Courant-Friedrichs-Lewy (CFL) condition is satisfied. Numerical experiments in twodimension (2D) and 3D are carried out and confirm our analysis, and the superconvergence in the discrete $H^{1}$ norm is found.
\end{abstract}

AMS subject classifications: 65M06, 65M12, 35L15, 78A48

Key words: Metamaterial Maxwell's equations, Yee scheme, non-uniform rectangular meshes, energy identities, stability.

\section{Introduction}

Metamaterials are artificial composite materials designed to exhibit exotic electromagnetic properties. The metamaterial with negative refraction index was first proposed by Veselago in 1968 [26] and constructed by Smith in 2000 [23,24], which has brought a new revolution in electromagnetic and material science. Since 2000, there are numerous reference sources on the study of metamaterials and their potential applications, such as, design of invisiblity cloak [8,22], sub-wavelength imaging [1,28], construction of perfect lens [25]. Matical side there has recently been increased interest in the understanding of the mathematical properties of metamaterial Maxwell's equations relevant to numerical analysis. For example, finite-difference time-domain (FDTD) methods [10,15-17], finite element methods $[11,13,29,30]$, and the monograph [12].

*Corresponding author.

Emails: 1318064638@qq.com (X. X. Bai), hxrui@sdu.edu.cn (H. X. Rui) 
Due to its efficiency and robustness, FDTD or Yee scheme, firstly introduced by Yee [31] in 1966, is still one of the most popular numerical methods in computational electromagnetics. The FDTD scheme uses central-difference approximations to the space and time partial derivatives at a fully staggered grid, and is second-order accurate in both time and space and easy to implement. For this aspect of theoretical study on the finite difference method for solving time-dependent Maxwell's equations, there are already excellent work in mathematical literature including FDTD scheme and related numerical methods, such as, the alternating direction implicit FDTD (ADI-FDTD) [20,32], the energy conserved splitting FDTD (EC-S-FDTD) [5, 20], the splitting FDTD (S-FDTD) [2], the splitting multi-symplectic method [7] etc. Among the above-mentioned methods, the stability and error estimates in the $L^{2}$ norm have been studied by the energy method. In 2011, Gao and Zhang [4] were firstly studied the important stability and convergence analysis in the $H^{1}$ norm of the FDTD scheme with PEC boundary condition on uniform meshes, and extended the similar results to other relevant numerical methods $[3,18]$.

Recently, the theoretical analysis of the Yee scheme on non-uniform meshes have attracted much attention. The rigorous error analysis of the Yee scheme on non-uniform rectangular meshes can be traced back 1994 by Monk and Süli [19]. They used the special structure of local errors to prove that the Yee scheme still has second-order convergent on a non-uniform mesh although the local truncation error is only of the first order. Remis [21] studied the stability condition of the Yee scheme for solving the Maxwell's equations in lossless medium on non-uniform meshes, by the eigenvalues of the FDTD iteration matrix. In 2016, Li and Shields [14] extended Monk and Süli's technique to give the superconvergence analysis of Yee scheme for solving Maxwell's equations in metamaterials on non-uniform meshes, and extended to an implicit scheme [27]. In these work, the energy method was used to study the stability and error estimates in the $L^{2}$ norm. However, no results is available for the import stability and convergence analysis in $H^{1}$ norm of the Yee scheme for metamaterial Maxwell's equations on non-uniform rectangular meshes.

Encouraged by the nice properties of the Yee scheme for Maxwell's equations in simple media on uniform meshes [4], in this paper, we study the stability and convergence of the Yee scheme for metamaterial Maxwell's equations on non-uniform rectangular meshes by a new energy method. This new method is motivated by the new energy identities of metamaterial Maxwell's equations established in this paper and is different from the usual one in $L^{2}$ norm (cf. $\left.[14,27]\right)$. By making use of this new energy method, we prove that the Yee scheme with the PEC boundary condition on non-uniform rectangular meshes is stable in the discrete $H^{1}$ norm when the CFL condition is satisfied. Numerical results are also presented to confirm the theoretical analysis. Moreover, the superconvergence phenomena are proved for solving metamaterial Maxwell's equations by the Yee scheme on non-uniform rectangular and cubic meshes. To our best knowledge, this is the first result for the important stability analysis in the discrete $H^{1}$ norm of the Yee scheme for solving metamaterial Maxwell's equations on non-uniform rectangular meshes.

The rest of the paper is organized as follows. In Section 2, the new energy identities 
of metamaterial Maxwell's equations in $H^{1}$ norm are proposed and the fully discrete Yee scheme on non-uniform rectangular meshes with some notations are given. We present detail analysis of the discrete stability in $L^{2}$ and $H^{1}$ norms of Yee scheme on non-uniform rectangular meshes in Section 3. In Section 4, numerical simulations results are demonstrated, and the superconvergence in the discrete $H^{1}$ norm is found. Besides, the classical example of backward wave propagation in metamaterials was shown. We conclude the paper in Section 5.

\section{Energy identities and the Yee scheme of metamaterial Maxwell's equations}

In this section, we first describe the problem of two-dimensional metamaterial Maxwell's equations with the PEC boundary condition in this paper. Then, we derive several new energy identities of metamaterial Maxwell's equations, which will be found helpful in study the Yee scheme in $H^{1}$ norm on non-uniform rectangular meshes.

\subsection{The metamaterial model}

Consider the following 2D Maxwell's equations in metamaterials [9]

$$
\left\{\begin{array}{l}
\epsilon_{0} \frac{\partial E_{x}}{\partial t}=\frac{\partial H_{z}}{\partial y}-J_{x} \\
\epsilon_{0} \frac{\partial E_{y}}{\partial t}=-\frac{\partial H_{z}}{\partial x}-J_{y} \\
\mu_{0} \frac{\partial H_{z}}{\partial t}=\frac{\partial E_{y}}{\partial x}-\frac{\partial E_{x}}{\partial y}-K_{z} \\
\frac{1}{\epsilon_{0} \omega_{p e}^{2}} \frac{\partial J_{x}}{\partial t}+\frac{\Gamma_{e}}{\epsilon_{0} \omega_{p e}^{2}} J_{x}=E_{x} \\
\frac{1}{\epsilon_{0} \omega_{p e}^{2}} \frac{\partial J_{y}}{\partial t}+\frac{\Gamma_{e}}{\epsilon_{0} \omega_{p e}^{2}} J_{y}=E_{y} \\
\frac{1}{\mu_{0} \omega_{p m}^{2}} \frac{\partial K_{z}}{\partial t}+\frac{\Gamma_{m}}{\mu_{0} \omega_{p m}^{2}} K_{z}=H_{z}
\end{array}\right.
$$

The PEC boundary condition is given by

$$
\mathbf{n} \times \mathbf{E}=\mathbf{0} \quad \text { on } \partial \Omega \text {, }
$$

and the initial conditions

$$
\mathbf{E}(\mathbf{x}, \mathbf{0})=\mathbf{E}^{\mathbf{0}}(\mathbf{x}), \quad H_{z}(\mathbf{x}, 0)=H_{z}^{0}(\mathbf{x}), \quad \mathbf{J}(\mathbf{x}, \mathbf{0})=\mathbf{J}^{\mathbf{0}}(\mathbf{x}), \quad K_{z}(\mathbf{x}, 0)=K_{z}^{0}(\mathbf{x}),
$$


where $\mathbf{E}=\left(E_{x}, E_{y}\right)^{T}, H_{z}, \mathbf{J}=\left(J_{x}, J_{y}\right)^{T}$ and $K_{z}$ denote the electric field, magnetic field, induced electric currents and induced magnetic currents vector, respectively. $\epsilon_{0}$ is the vacuum permittivity, $\mu_{0}$ is the vacuum permeability, $\omega_{p e}, \omega_{p m}, \Gamma_{e}$ and $\Gamma_{m}$, respectively, are electric plasma frequency, magnetic plasma frequency, electric damping frequency and magnetic damping frequency. $\mathbf{n}$ represents the unit exterior normal vector to the boundary of the spatial domains $\Omega=[a, b] \times[c, d]$. The initial conditions $\mathbf{E}^{0}, H_{z}^{0}, \mathbf{J}^{0}, K_{z}^{0}$ denote some given functions.

\subsection{Energy identities of Maxwell's equations in metamaterial}

The following Lemma 2.1 which is the well-known Poynting theorem, its proof can be seen in [14].

Lemma 2.1. Let $\mathbf{E}(t)=\left(E_{x}(t, x, y), E_{y}(t, x, y)\right), H_{z}(t)=H_{z}(t, x, y), \mathbf{J}(t)=\left(J_{x}(t, x, y), J_{y}(t, x, y)\right)$, $K_{z}(t)=K_{z}(t, x, y)$ be the solution to the problem (2.1)-(2.8). Then for any $t \in(0, T]$,

$$
\begin{aligned}
& \|\mathbf{E}(t)\|^{2}+\left\|H_{z}(t)\right\|^{2}+\|\mathbf{J}(t)\|^{2}+\left\|K_{z}(t)\right\|^{2}+2 \int_{0}^{T} \Gamma_{e}\|\mathbf{J}(\tau)\|^{2}+\Gamma_{m}\left\|K_{z}(\tau)\right\|^{2} d \tau \\
= & \|\mathbf{E}(0)\|^{2}+\left\|H_{z}(0)\right\|^{2}+\|\mathbf{J}(0)\|^{2}+\left\|K_{z}(0)\right\|^{2},
\end{aligned}
$$

where $\|\cdot\|$ denotes the $L^{2}$ norm with the weight. For example, in (2.9),

$$
\begin{array}{ll}
\|\mathbf{E}(t)\|^{2}=\left\|E_{x}(t)\right\|^{2}+\left\|E_{y}(t)\right\|^{2}, & \left\|E_{x}(t)\right\|^{2}=\int_{a}^{b} \int_{c}^{d} \epsilon_{0}\left|E_{x}(x, y, t)\right|^{2} d y d x, \\
\|\mathbf{J}(t)\|^{2}=\left\|J_{x}(t)\right\|^{2}+\left\|J_{y}(t)\right\|^{2}, & \left\|J_{x}(t)\right\|^{2}=\int_{a}^{b} \int_{c}^{d} \frac{1}{\epsilon_{0} \omega_{p e}^{2}}\left|J_{x}(x, y, t)\right|^{2} d y d x .
\end{array}
$$

Using similar techniques as in [4], we can obtain the following new energy identities.

Theorem 2.1. Let the $\mathbf{E}(t), H_{z}(t), \mathbf{J}(t), K_{z}(t)$ be the solution to the problem (2.1)-(2.8), and possesses the following regularity property:

$$
\begin{aligned}
& E_{x}, E_{y}, H_{z} \in C^{1}\left([0, T], C^{2}(\bar{\Omega})\right), \\
& J_{x}, J_{y}, K_{z} \in C^{1}\left([0, T], C^{1}(\bar{\Omega})\right) .
\end{aligned}
$$

Then for $u=x, y$,

$$
\begin{aligned}
& \left\|\frac{\partial \mathbf{E}(t)}{\partial u}\right\|^{2}+\left\|\frac{\partial H_{z}(t)}{\partial u}\right\|^{2}+\left\|\frac{\partial \mathbf{J}(t)}{\partial u}\right\|^{2}+\left\|\frac{\partial K_{z}(t)}{\partial u}\right\|^{2}+2 \int_{0}^{t} \Gamma_{e}\left\|\frac{\partial \mathbf{J}(\tau)}{\partial u}\right\|^{2}+\Gamma_{m}\left\|\frac{\partial K_{z}(\tau)}{\partial u}\right\|^{2} d \tau \\
& =\left\|\frac{\partial \mathbf{E}(0)}{\partial u}\right\|^{2}+\left\|\frac{\partial H_{z}(0)}{\partial u}\right\|^{2}+\left\|\frac{\partial \mathbf{J}(0)}{\partial u}\right\|^{2}+\left\|\frac{\partial K_{z}(0)}{\partial u}\right\|^{2} \\
& \|\mathbf{E}(t)\|_{1}^{2}+\left\|H_{z}(t)\right\|_{1}^{2}+\|\mathbf{J}(t)\|_{1}^{2}+\left\|K_{z}(t)\right\|_{1}^{2}+2 \int_{0}^{t} \Gamma_{e}\|\mathbf{J}(\tau)\|_{1}^{2}+\Gamma_{m}\left\|K_{z}(\tau)\right\|_{1}^{2} d \tau \\
& =\|\mathbf{E}(0)\|_{1}^{2}+\left\|H_{z}(0)\right\|_{1}^{2}+\|\mathbf{J}(0)\|_{1}^{2}+\left\|K_{z}(0)\right\|_{1}^{2}
\end{aligned}
$$


where $\|\cdot\|_{1}$ is the $H^{1}(\Omega)$ norm, defined by

$$
\|f\|_{1}^{2}=\|f\|^{2}+\left\|\frac{\partial f}{\partial x}\right\|^{2}+\left\|\frac{\partial f}{\partial y}\right\|^{2}
$$

Proof. We only prove (2.10a) with $u=x$. The other cases with $u=y$ can be obtained similarly.

Differentiating each of Eqs. (2.1)-(2.6) with respect to $x$, we have

$$
\left\{\begin{array}{l}
\epsilon_{0} \frac{\partial^{2} E_{x}}{\partial x \partial t}=\frac{\partial^{2} H_{z}}{\partial x \partial y}-\frac{\partial J_{x}}{\partial x} \\
\epsilon_{0} \frac{\partial^{2} E_{y}}{\partial x \partial t}=-\frac{\partial^{2} H_{z}}{\partial^{2} x}-\frac{\partial J_{y}}{\partial x} \\
\mu_{0} \frac{\partial^{2} H_{z}}{\partial x \partial t}=\frac{\partial^{2} E_{y}}{\partial^{2} x}-\frac{\partial^{2} E_{x}}{\partial x \partial y}-\frac{\partial K_{z}}{\partial x} \\
\frac{1}{\epsilon_{0} \omega_{p e}^{2}} \frac{\partial^{2} J_{x}}{\partial x \partial t}+\frac{\Gamma_{e}}{\epsilon_{0} \omega_{p e}^{2}} \frac{\partial J_{x}}{\partial x}=\frac{\partial E_{x}}{\partial x} \\
\frac{1}{\epsilon_{0} \omega_{p e}^{2}} \frac{\partial^{2} J_{y}}{\partial x \partial t}+\frac{\Gamma_{e}}{\epsilon_{0} \omega_{p e}^{2}} \frac{\partial J_{y}}{\partial x}=\frac{\partial E_{y}}{\partial x} \\
\frac{1}{\mu_{0} \omega_{p m}^{2}} \frac{\partial^{2} K_{z}}{\partial x \partial t}+\frac{\Gamma_{m}}{\mu_{0} \omega_{p m}^{2}} \frac{\partial K_{z}}{\partial x}=\frac{\partial H_{z}}{\partial x}
\end{array}\right.
$$

Multiplying (2.11) by $\frac{\partial E_{x}}{\partial x},(2.12)$ by $\frac{\partial E_{y}}{\partial x},(2.13)$ by $\frac{\partial H_{z}}{\partial x},(2.14)$ by $\frac{\partial J_{x}}{\partial x},(2.15)$ by $\frac{\partial J_{y}}{\partial x},(2.16)$ by $\frac{\partial K_{z}}{\partial x}$, then summing the six product equations up over $\Omega$, we obtain

$$
\begin{aligned}
& \frac{1}{2} \frac{d}{d t}\left(\left\|\frac{\partial E_{x}}{\partial x}\right\|^{2}+\left\|\frac{\partial E_{y}}{\partial x}\right\|^{2}+\left\|\frac{\partial H_{z}}{\partial x}\right\|^{2}+\left\|\frac{\partial J_{x}}{\partial x}\right\|^{2}+\left\|\frac{\partial J_{y}}{\partial x}\right\|^{2}+\left\|\frac{\partial K_{z}}{\partial x}\right\|^{2}\right) \\
& \quad+\Gamma_{e}\left(\left\|\frac{\partial J_{x}(\tau)}{\partial x}\right\|^{2}+\left\|\frac{\partial J_{y}(\tau)}{\partial x}\right\|^{2}\right)+\Gamma_{m}\left\|\frac{\partial K_{z}(\tau)}{\partial x}\right\|^{2}=r(t)
\end{aligned}
$$

where

$$
r(t)=\int_{a}^{b} \int_{c}^{d}\left(\frac{\partial^{2} H_{z}}{\partial x \partial y} \frac{\partial E_{x}}{\partial x}-\frac{\partial^{2} H_{z}}{\partial^{2} x} \frac{\partial E_{y}}{\partial x}+\frac{\partial^{2} E_{y}}{\partial^{2} x} \frac{\partial H_{z}}{\partial x}-\frac{\partial^{2} E_{x}}{\partial x \partial y} \frac{\partial H_{z}}{\partial x}\right) .
$$

From the PEC boundary conditions (2.7) and the domain $\Omega=[a, b] \times[c, d]$, it follows that

$$
E_{x}(x, c)=E_{x}(x, d)=0, \quad E_{y}(a, y)=E_{y}(b, y)=0,
$$

thus

$$
\begin{aligned}
& \frac{\partial E_{x}(x, c)}{\partial x}=\frac{\partial E_{x}(x, d)}{\partial x}=0 \\
& \frac{\partial E_{y}(a, y)}{\partial y}=\frac{\partial E_{y}(b, y)}{\partial y}=0 .
\end{aligned}
$$


Using integration by parts and Eq. (2.20a), we have

$$
\begin{aligned}
& \frac{1}{2} \frac{d}{d t}\left(\left\|\frac{\partial E_{x}}{\partial x}\right\|^{2}+\left\|\frac{\partial E_{y}}{\partial x}\right\|^{2}+\left\|\frac{\partial H_{z}}{\partial x}\right\|^{2}+\left\|\frac{\partial J_{x}}{\partial x}\right\|^{2}+\left\|\frac{\partial J_{y}}{\partial x}\right\|^{2}+\left\|\frac{\partial K_{z}}{\partial x}\right\|^{2}\right) \\
& \quad+\Gamma_{e}\left\|\frac{\partial \mathbf{J}(\tau)}{\partial u}\right\|^{2}+\Gamma_{m}\left\|\frac{\partial K_{z}(\tau)}{\partial u}\right\|^{2}=r(t),
\end{aligned}
$$

where

$$
r(t)=\int_{c}^{d} \frac{\partial E_{y}}{\partial x}(b, y, t) \frac{\partial H_{z}}{\partial x}(b, y, t)-\frac{\partial E_{y}}{\partial x}(a, y, t) \frac{\partial H_{z}}{\partial x}(a, y, t) d y .
$$

Let $\hat{x}=a$ or $b$, from (2.2) and the PEC boundary condition (2.7), we have

$$
\begin{aligned}
& \left(\frac{\partial E_{y}}{\partial x} \frac{\partial H_{z}}{\partial x}\right)(\hat{x}, y, t)=\lim _{x \rightarrow \hat{x}}\left(\frac{\partial E_{y}}{\partial x} \frac{\partial H_{z}}{\partial x}\right)(x, y, t) \\
= & -\lim _{x \rightarrow \hat{x}} \frac{\partial E_{y}}{\partial x}(x, y, t)\left(\epsilon_{0} \frac{\partial E_{y}}{\partial t}+J_{y}\right)(x, y, t)=0 .
\end{aligned}
$$

Thus, $r(t)=0$.

Then, by integrating (2.21) with respect to time over $[0, t],(0 \leq t \leq T)$, we get

$$
\begin{aligned}
& \left\|\frac{\partial \mathrm{E}(t)}{\partial x}\right\|^{2}+\left\|\frac{\partial H_{z}(t)}{\partial x}\right\|^{2}+\left\|\frac{\partial \mathbf{J}(t)}{\partial x}\right\|^{2}+\left\|\frac{\partial K_{z}(t)}{\partial x}\right\|^{2}+2 \int_{0}^{t} \Gamma_{e}\left\|\frac{\partial \mathbf{J}(\tau)}{\partial x}\right\|^{2}+\Gamma_{m}\left\|\frac{\partial K_{z}(\tau)}{\partial x}\right\|^{2} d \tau \\
= & \left\|\frac{\partial \mathrm{E}(0)}{\partial x}\right\|^{2}+\left\|\frac{\partial H_{z}(0)}{\partial x}\right\|^{2}+\left\|\frac{\partial \mathbf{J}(0)}{\partial x}\right\|^{2}+\left\|\frac{\partial K_{z}(0)}{\partial x}\right\|^{2} .
\end{aligned}
$$

We complete the proof of (2.10a), with $u=x$. Combining (2.9) and (2.10a), Eq. (2.10b) holds.

\subsection{The fully discrete Yee scheme on non-uniform rectangular meshes}

The rectangular domain $[a, b] \times[c, d]$ is partitioned by a non-uniform rectangular grid as follows:

$$
a=x_{0}<x_{1}<\cdots<x_{I}=b, \quad c=y_{0}<y_{1}<\cdots<y_{J}=d .
$$

Denote

$$
x_{i+\frac{1}{2}}=\frac{x_{i}+x_{i+1}}{2}, \quad i=0, \cdots, I-1, \quad y_{j+\frac{1}{2}}=\frac{y_{j}+y_{j+1}}{2}, \quad j=0, \cdots, J-1 .
$$

For convenience, denote the following mesh step sizes $h_{i}, k_{j}, h_{i+\frac{1}{2}}, k_{j+\frac{1}{2}}$

$$
\begin{array}{ll}
h_{i}=x_{i+\frac{1}{2}}-x_{i-\frac{1}{2}}, & h_{i+\frac{1}{2}}=x_{i+1}-x_{i}, \\
k_{j}=y_{j+\frac{1}{2}}-y_{j-\frac{1}{2}}, & k_{j+\frac{1}{2}}=y_{j+1}-y_{j}, \\
h_{\min }=\min \left\{h_{i}, h_{i+\frac{1}{2}}\right\}, & k_{\min }=\min \left\{k_{j}, k_{j+\frac{1}{2}}\right\} .
\end{array}
$$


Denote $\Delta t$ as the time step size, $N_{t} \Delta t=T$. For $\alpha=i, i+\frac{1}{2}, \beta=j, j+\frac{1}{2}$ and $n=m, m+\frac{1}{2}$, where $i=0,1, \cdots, I-1, j=0,1, \cdots, J-1, m=0,1, \cdots, N_{t}$ with positive integers $I, J$ and $N_{t}$, then for a grid function $f_{\alpha, \beta}^{n}=f\left(x_{\alpha}, y_{\beta}, t^{n}\right)$, define

$$
\delta_{x} f_{\alpha, \beta}^{n}=\frac{f_{\alpha+\frac{1}{2}, \beta}^{n}-f_{\alpha-\frac{1}{2}, \beta}^{n}}{h_{\alpha}}, \quad \delta_{y} f_{\alpha, \beta}^{n}=\frac{f_{\alpha, \beta+\frac{1}{2}}^{n}-f_{\alpha, \beta-\frac{1}{2}}^{n}}{k_{\beta}} .
$$

With the above preparation, we can have the following fully-discrete Yee scheme:

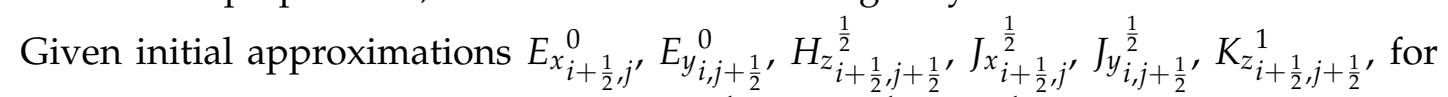
any $0 \leq n \leq N_{t}-1$, solve $E_{x_{i+\frac{1}{2}, j}^{N_{t}}}^{N_{t}}, E_{y_{i, j+\frac{1}{2}}}^{N_{t}}, H_{z_{i+\frac{1}{2}, j+\frac{1}{2}}}^{N_{t}+\frac{1}{2}} J_{x_{i+\frac{1}{2}, j}}^{N_{t}+\frac{1}{2}}, J_{y_{i, j+\frac{1}{2}}}^{N_{t}+\frac{1}{2}}, K_{z_{i+\frac{1}{2}, j+\frac{1}{2}}}^{N_{t}+1}$ from:

$$
\left\{\begin{array}{l}
\epsilon_{0} \frac{E_{x}^{n+1}-E_{x}^{n}}{\Delta t}=\delta_{y} H_{z}^{n+\frac{1}{2}}-\left.J_{x}^{n+\frac{1}{2}}\right|_{i+\frac{1}{2}, j^{\prime}} \\
\epsilon_{0} \frac{E_{y}^{n+1}-E_{y}^{n}}{\Delta t}=-\delta_{x} H_{z}^{n+\frac{1}{2}}-\left.J_{y}^{n+\frac{1}{2}}\right|_{i, j+\frac{1}{2} \prime^{\prime}} \\
\mu_{0} \frac{H_{z}^{n+\frac{3}{2}}-H_{z}^{n+\frac{1}{2}}}{\Delta t}=\delta_{y} E_{x}^{n+1}-\delta_{x} E_{y}^{n+1}-\left.K_{z}^{n+1}\right|_{i+\frac{1}{2}, j+\frac{1}{2}}, \\
\frac{1}{\epsilon_{0} \omega_{p e}^{2}} \frac{J_{x}^{n+\frac{3}{2}}-J_{x}^{n+\frac{1}{2}}}{\Delta t}+\frac{\Gamma_{e}}{\epsilon_{0} \omega_{p e}^{2}} \frac{J_{x}^{n+\frac{3}{2}}+J_{x}^{n+\frac{1}{2}}}{2}=\left.E_{x}^{n+1}\right|_{i+\frac{1}{2}, j^{\prime}} \\
\frac{1}{\epsilon_{0} \omega_{p e}^{2}} \frac{J_{y}^{n+\frac{3}{2}}-J_{y}^{n+\frac{1}{2}}}{\Delta t}+\frac{\Gamma_{e}}{\epsilon_{0} \omega_{p e}^{2}} \frac{J_{y}^{n+\frac{3}{2}}+J_{y}^{n+\frac{1}{2}}}{2}=\left.E_{y}^{n+1}\right|_{i, j+\frac{1}{2}}, \\
\frac{1}{\mu_{0} \omega_{p m}^{2}} \frac{K_{z}^{n+2}-K_{y}^{n+1}}{\Delta t}+\frac{\Gamma_{m}}{\mu_{0} \omega_{p m}^{2}} \frac{K_{z}^{n+2}+K_{z}^{n+1}}{2}=\left.H_{z}^{n+\frac{3}{2}}\right|_{i+\frac{1}{2}, j+\frac{1}{2}} .
\end{array}\right.
$$

The PEC boundary condition

$$
E_{x_{i+\frac{1}{2}, 0}^{n}}^{n}=E_{x_{i+\frac{1}{2}, J}^{n}}^{n}=E_{y_{0, j+\frac{1}{2}}}^{n}=E_{y_{I, j+\frac{1}{2}}}^{n}=0, \quad n=0,1, \cdots, N_{t} .
$$

The initial conditions which are obtained by imposing the initial conditions (2.8) at $t=0$,

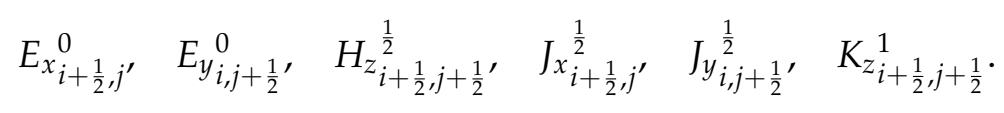

Remark 2.1. From Eqs. (2.4), (2.5) and the PEC boundary condition (2.30), we can easily get

$$
J_{x_{i+\frac{1}{2}, 0}^{n+\frac{1}{2}}}^{n+} J_{x_{i+\frac{1}{2}, J}^{n+\frac{1}{2}}}^{n+} J_{y_{0, j+\frac{1}{2}}^{n+\frac{1}{2}}}^{n+J_{y^{\prime}, j+\frac{1}{2}}^{n+\frac{1}{2}}}=0, \quad n=0,1, \cdots, N_{t} .
$$




\section{Stability of the Yee scheme on non-uniform rectangular meshes}

In this section, we investigate the stability of the Yee scheme on non-uniform rectangular meshes in the discrete $L^{2}$ and $H^{1}$ norms, by the similar techniques as in the last section.

\subsection{Discrete mesh-dependent energy norms}

We define the following discrete mesh-dependent energy norms. For a grid function $V_{\alpha, \beta}$, where $\alpha=i$ or $i+\frac{1}{2}, \beta=j$ or $j+\frac{1}{2}$,

$$
\begin{array}{ll}
\left\|V_{x}\right\|_{E_{x}}^{2}=\sum_{i=0}^{I-1} \sum_{j=1}^{J-1} \epsilon_{0}\left(V_{x_{i+\frac{1}{2}, j}}\right)^{2} h_{i+\frac{1}{2}} k_{j}, & \left\|V_{y}\right\|_{E_{y}}^{2}=\sum_{i=1}^{I-1} \sum_{j=0}^{J-1} \epsilon_{0}\left(V_{y_{i, j+\frac{1}{2}}}\right)^{2} h_{i} k_{j+\frac{1}{2}}, \\
\left\|V_{x}\right\|_{J_{x}}^{2}=\sum_{i=0}^{I-1} \sum_{j=1}^{J-1} \frac{1}{\epsilon_{0} \omega_{p e}^{2}}\left(V_{x_{i+\frac{1}{2}, j}}\right)^{2} h_{i+\frac{1}{2}} k_{j}, & \left\|V_{z}\right\|_{K_{z}}^{2}=\sum_{i=0}^{I-1} \sum_{j=0}^{J-1} \frac{1}{\mu_{0} \omega_{p m}^{2}}\left(V_{z_{i+\frac{1}{2}, j+\frac{1}{2}}}\right)^{2} h_{i+\frac{1}{2}} k_{j+\frac{1}{2}}, \\
\left\|V_{z}\right\|_{H_{z}}^{2}=\sum_{i=0}^{I-1 J-1} \sum_{j=0}^{I-1} \mu_{0}\left(V_{z_{i+\frac{1}{2}, j+\frac{1}{2}}}\right)^{2} h_{i+\frac{1}{2}} k_{j+\frac{1}{2}}, & \left\|\delta_{x} V_{x}\right\|_{\delta_{x} E_{x}}^{2}=\sum_{i=1}^{I-1 J-1} \sum_{j=1} \epsilon_{0}\left(\delta_{x} V_{x_{i, j}}\right)^{2} h_{i} k_{j}, \\
\left\|\delta_{x} V_{y}\right\|_{\delta_{x} E_{y}}^{2}=\sum_{i=1}^{I-2 J-1} \sum_{j=0} \epsilon_{0}\left(\delta_{x} V_{y_{i+\frac{1}{2}, j+\frac{1}{2}}}\right)^{2} h_{i+\frac{1}{2}} k_{j+\frac{1}{2}}, & \left\|\delta_{x} V_{z}\right\|_{\delta_{x} H_{z}}^{2}=\sum_{i=1}^{I-1 J-1} \sum_{j=0} \mu_{0}\left(\delta_{x} V_{z_{i, j+\frac{1}{2}}}\right)^{2} h_{i} k_{j+\frac{1}{2}}, \\
|V|_{x}^{2}=\sum_{i=0}^{I-1} \epsilon_{0}\left(\left(V_{i, 1}\right)^{2} \frac{h_{i}}{k_{\frac{1}{2}}}+\left(V_{i, J-1}\right)^{2} \frac{h_{i}}{k_{J-\frac{1}{2}}}\right), & |V|_{y}^{2}=\sum_{j=0}^{J-1} \epsilon_{0}\left(\left(V_{1, j}\right)^{2} \frac{k_{j}}{h_{\frac{1}{2}}}+\left(V_{I-1, j}\right)^{2} \frac{k_{j}}{h_{I-\frac{1}{2}}}\right) .
\end{array}
$$

The norms $\|\cdot\|_{J_{y}},\|\cdot\|_{\delta_{x} J_{x}},\|\cdot\|_{\delta_{x} J_{y}},\|\cdot\|_{\delta_{x} K_{z}},\|\cdot\|_{\delta_{y} E_{x}},\|\cdot\|_{\delta_{y} E_{y}},\|\cdot\|_{\delta_{y} H_{z}},\|\cdot\|_{\delta_{y} J_{x}},\|\cdot\|_{\delta_{y} J_{y}},\|\cdot\|_{\delta_{y} K_{z}}$ can be similarly defined by changing the indices $i, j$. In these norms, the subscripts mean that the sum is taken over the sets of the spatial indices.

\subsection{Stability of the Yee scheme in the discrete $H^{1}$ semi-norm}

The following lemma will be used in the following analysis. It can be easily proved by using summation by parts and the PEC boundary condition (2.30).

Lemma 3.1. Let $E_{x_{i+\frac{1}{2}, j}^{n}}^{n} E_{y_{i, j+\frac{1}{2}}^{n}}^{n}, H_{z_{i+\frac{1}{2}, j+\frac{1}{2}}^{m}}$ be the solution to the fully discrete Yee scheme with the PEC boundary condition (2.30), then for $m=n+\frac{1}{2}$ or $n-\frac{1}{2}$ with $n \geq 1$, we have

$$
\left.\sum_{i=1}^{I-1} \sum_{j=0}^{J-1} \delta_{x} \delta_{y} E_{x}^{n} \cdot \delta_{x} H_{z}^{m}\right|_{i, j+\frac{1}{2}} h_{i} k_{j+\frac{1}{2}}=-\left.\sum_{i=1}^{I-1} \sum_{j=1}^{J-1} \delta_{x} E_{x}^{n} \cdot \delta_{x} \delta_{y} H_{z}^{m}\right|_{i, j} h_{i} k_{j}
$$




$$
\begin{aligned}
& \left.\sum_{i=1}^{I-1} \sum_{j=0}^{J-1} \delta_{x} \delta_{x} E_{y}^{n} \cdot \delta_{x} H_{z}^{m}\right|_{i, j+\frac{1}{2}} h_{i} k_{j+\frac{1}{2}} \\
& =-\left.\sum_{i=1}^{I-2 J-1} \sum_{j=0} \delta_{x} E_{y}^{n} \cdot \delta_{x} \delta_{x} H_{z}^{m}\right|_{i+\frac{1}{2}, j+\frac{1}{2}} h_{i+\frac{1}{2}} k_{j+\frac{1}{2}} \\
& \quad-\left.\sum_{j=0}^{J-1} E_{y}^{n} \cdot \delta_{x} H_{z}^{m}\right|_{1, j+\frac{1}{2}} \frac{k_{j+\frac{1}{2}}}{h_{\frac{1}{2}}}-\left.\sum_{j=0}^{J-1} E_{y}^{n} \cdot \delta_{x} H_{z}^{m}\right|_{I-1, j+\frac{1}{2}} \frac{k_{j+\frac{1}{2}}}{h_{I-\frac{1}{2}}}, \\
& \left.\sum_{i=0}^{I-1 J-1} \sum_{j=1}^{J-1} \delta_{y} \delta_{y} E_{x}^{n} \cdot \delta_{y} H_{z}^{m}\right|_{i+\frac{1}{2}, j} h_{i+\frac{1}{2}} k_{j} \\
& =-\left.\sum_{i=0}^{I-1 J-2} \sum_{j=1} \delta_{y} E_{x}^{n} \cdot \delta_{y} \delta_{y} H_{z}^{m}\right|_{i+\frac{1}{2}, j+\frac{1}{2}} h_{i+\frac{1}{2}} k_{j+\frac{1}{2}} \\
& \quad-\left.\sum_{i=0}^{I-1} E_{x}^{n} \cdot \delta_{y} H_{z}^{m}\right|_{i+\frac{1}{2}, 1} \frac{h_{i+\frac{1}{2}}}{k_{\frac{1}{2}}}-\left.\sum_{i=0}^{I-1} E_{x}^{n} \cdot \delta_{y} H_{z}^{m}\right|_{i+\frac{1}{2}, J-1} \frac{h_{i+\frac{1}{2}}}{k_{J-\frac{1}{2}}} \\
& \left.\sum_{i=0}^{I-1 J-1} \sum_{j=1}^{J-1} \delta_{y} \delta_{x} E_{y}^{n} \cdot \delta_{y} H_{z}^{m}\right|_{i+\frac{1}{2}, j} h_{i+\frac{1}{2}} k_{j}=-\left.\sum_{i=1}^{I-1 J-1} \sum_{j=1} \delta_{y} E_{y}^{n} \cdot \delta_{x} \delta_{y} H_{z}^{m}\right|_{i, j} h_{i} k_{j} .
\end{aligned}
$$

Theorem 3.1. For $n \geq 0$, let $E_{x_{i+\frac{1}{2}, j}}^{n}, E_{y_{i, j+\frac{1}{2}}}^{n}, H_{z_{i+\frac{1}{2}, j+\frac{1}{2}}^{n+\frac{1}{2}}}, J_{x_{i+\frac{1}{2}, j^{\prime}}}^{n+\frac{1}{2}} J_{y_{i, j+\frac{1}{2}}}^{n+\frac{1}{2}}, K_{z_{i+\frac{1}{2}, j+\frac{1}{2}}^{n+1}}^{n}$ be the solution to the fully discrete Yee scheme (2.24)-(2.29) with the PEC boundary condition (2.30), and possesses the following regularity property:

$$
\begin{aligned}
& E_{x}, E_{y}, H_{z} \in C^{1}\left([0, T], C^{2}(\bar{\Omega})\right), \\
& J_{x}, J_{y}, K_{z} \in C^{1}\left([0, T], C^{1}(\bar{\Omega})\right) .
\end{aligned}
$$

If the CFL condition

$$
\Delta t<\min \left\{\frac{\sqrt{\mu_{0} \epsilon_{0}}}{2} \sqrt{\frac{h_{\min }^{2} k_{\min }^{2}}{h_{\min }^{2}+k_{\min }^{2}}}, \frac{\sqrt{2}}{\sqrt{3} \omega_{p e}}, \frac{1}{\omega_{p m}}\right\}
$$

is satisfied, then the following estimate holds

$$
\begin{aligned}
& \left|\left(E_{x}^{n}, E_{y}^{n}\right)\right|_{1}^{2}+\left|\left(J_{x}^{n+\frac{1}{2}}, J_{y}^{n+\frac{1}{2}}\right)\right|_{1}^{2}+\left|H_{z}^{n+\frac{1}{2}}\right|_{1}^{2}+\left|K_{z}^{n+1}\right|_{1}^{2} \\
\leq & C\left\{\left|\left(E_{x}^{0}, E_{y}^{0}\right)\right|_{1}^{2}+\left|\left(J_{x}^{\frac{1}{2}}, J_{y}^{\frac{1}{2}}\right)\right|_{1}^{2}+\left|H_{z}^{\frac{1}{2}}\right|_{1}^{2}+\left|K_{z}^{1}\right|_{1}^{2}\right\}
\end{aligned}
$$

for $n \geq 1$ where $C$ is a constant independent of $n$ and for $n \geq 0$, the discrete $H^{1}$ semi-norm is defined as

$$
\left|\left(E_{x}^{n}, E_{y}^{n}\right)\right|_{1}^{2}=\left\|\delta_{x} E_{x}^{n}\right\|_{\delta_{x} E_{x}}^{2}+\left\|\delta_{x} E_{y}^{n}\right\|_{\delta_{x} E_{y}}^{2}+\left\|\delta_{y} E_{x}^{n}\right\|_{\delta_{y} E_{x}}^{2}+\left\|\delta_{y} E_{y}^{n}\right\|_{\delta_{y} E_{y}}^{2}+\left|E_{x}^{n}\right|_{x}^{2}+\left|E_{y}^{n}\right|_{y}^{2}
$$




$$
\begin{aligned}
& \left|\left(J_{x}^{n+\frac{1}{2}}, J_{y}^{n+\frac{1}{2}}\right)\right|_{1}^{2}=\left\|\delta_{x} J_{x}^{n+\frac{1}{2}}\right\|_{\delta_{x} J_{x}}^{2}+\left\|\delta_{x} J_{y}^{n+\frac{1}{2}}\right\|_{\delta_{x} J_{y}}^{2}+\left\|\delta_{y} J_{x}^{n+\frac{1}{2}}\right\|_{\delta_{y} J_{x}}^{2}+\left\|\delta_{y} J_{y}^{n+\frac{1}{2}}\right\|_{\delta_{y} J_{y}}^{2}, \\
& \left|H_{z}^{n+\frac{1}{2}}\right|_{1}^{2}=\left\|\delta_{x} H_{z}^{n+\frac{1}{2}}\right\|_{\delta_{x} H_{z}}^{2}+\left\|\delta_{y} H_{z}^{n+\frac{1}{2}}\right\|_{\delta_{y} H_{z}}^{2}, \quad\left|K_{z}^{n+1}\right|_{1}^{2}=\left\|\delta_{x} K_{z}^{n+1}\right\|_{\delta_{x} K_{z}}^{2}+\left\|\delta_{y} K_{z}^{n+1}\right\|_{\delta_{y} K_{z}}^{2} .
\end{aligned}
$$

Proof. Applying the difference operator $\delta_{x}$ to the equations in the fully discrete Yee scheme(2.24)-(2.29) gives the $\delta_{x}$-Yee scheme:

$$
\begin{aligned}
& \epsilon_{0} \frac{\delta_{x} E_{x}^{n+1}-\delta_{x} E_{x}^{n}}{\Delta t}=\delta_{x} \delta_{y} H_{z}^{n+\frac{1}{2}}-\left.\delta_{x} J_{x}^{n+\frac{1}{2}}\right|_{i, j}, \\
& \epsilon_{0} \frac{\delta_{x} E_{y}^{n+1}-\delta_{x} E_{y}^{n}}{\Delta t}=-\delta_{x} \delta_{x} H_{z}^{n+\frac{1}{2}}-\left.\delta_{x} J_{y}^{n+\frac{1}{2}}\right|_{i+\frac{1}{2}, j+\frac{1}{2}} \\
& \mu_{0} \frac{\delta_{x} H_{z}^{n+\frac{3}{2}}-\delta_{x} H_{z}^{n+\frac{1}{2}}}{\Delta t}=\delta_{x} \delta_{y} E_{x}^{n+1}-\delta_{x} \delta_{x} E_{y}^{n+1}-\left.\delta_{x} K_{z}^{n+1}\right|_{i, j+\frac{1}{2} \prime^{\prime}} \\
& \frac{1}{\epsilon_{0} \omega_{p e}^{2}} \frac{\delta_{x} J_{x}^{n+\frac{3}{2}}-\delta_{x} J_{x}^{n+\frac{1}{2}}}{\Delta t}+\frac{\Gamma_{e}}{\epsilon_{0} \omega_{p e}^{2}} \frac{\delta_{x} J_{x}^{n+\frac{3}{2}}+\delta_{x} J_{x}^{n+\frac{1}{2}}}{2}=\left.\delta_{x} E_{x}^{n+1}\right|_{i, j}, \\
& \frac{1}{\epsilon_{0} \omega_{p e}^{2}} \frac{\delta_{x} J_{y}^{n+\frac{3}{2}}-\delta_{x} J_{y}^{n+\frac{1}{2}}}{\Delta t}+\frac{\Gamma_{e}}{\epsilon_{0} \omega_{p e}^{2}} \frac{\delta_{x} J_{y}^{n+\frac{3}{2}}+\delta_{x} J_{y}^{n+\frac{1}{2}}}{2}=\left.\delta_{x} E_{y}^{n+1}\right|_{i+\frac{1}{2}, j+\frac{1}{2}} \\
& \frac{1}{\mu_{0} \omega_{p m}^{2}} \frac{\delta_{x} K_{z}^{n+2}-\delta_{x} K_{y}^{n+1}}{\Delta t}+\frac{\Gamma_{m}}{\mu_{0} \omega_{p m}^{2}} \frac{\delta_{x} K_{z}^{n+2}+\delta_{x} K_{z}^{n+1}}{2}=\left.\delta_{x} H_{z}^{n+\frac{3}{2}}\right|_{i, j+\frac{1}{2}} .
\end{aligned}
$$

Note that the subscript $i, j$ in each equation and its range are changed after the application by $\delta_{x}$.

Multiplying both sides of Eqs. (3.2a)-(3.2f) by $\left.\Delta t\left(\delta_{x} E_{x}^{n+1}+\delta_{x} E_{x}^{n}\right)\right|_{i, j} h_{i} k_{j}, \Delta t\left(\delta_{x} E_{y}^{n+1}+\right.$ $\left.\delta_{x} E_{y}^{n}\right)\left.\right|_{i+\frac{1}{2}, j} h_{i+\frac{1}{2}} k_{j},\left.\quad \Delta t\left(\delta_{x} H_{z}^{n+\frac{3}{2}}+\delta_{x} H_{z}^{n+\frac{1}{2}}\right)\right|_{i, j+\frac{1}{2}} h_{i} k_{j+\frac{1}{2}},\left.\quad\left(\delta_{x} J_{x}^{n+\frac{3}{2}}+\delta_{x} J_{x}^{n+\frac{1}{2}}\right)\right|_{i, j} h_{i} k_{j}, \quad\left(\delta_{x} J_{y}^{n+\frac{3}{2}}+\right.$ $\left.\delta_{x} J_{y}^{n+\frac{1}{2}}\right)\left.\right|_{i+\frac{1}{2}, j} h_{i+\frac{1}{2}} k_{j},\left.\left(\delta_{x} K_{z}^{n+2}+\delta_{x} K_{z}^{n+1}\right)\right|_{i, j+\frac{1}{2}} h_{i} k_{j+\frac{1}{2}}$, respectively, and summing them up over $i, j$ in their valid ranges. By adding the six equations together, we obtain the sum of the left hand side (LHS) as

$$
\begin{aligned}
\text { LHS }= & \left.\sum_{i=1}^{I-1} \sum_{j=1}^{J-1} \epsilon_{0}\left(\left(\delta_{x} E_{x}^{n+1}\right)^{2}-\left(\delta_{x} E_{x}^{n}\right)^{2}\right)\right|_{i, j} h_{i} k_{j} \\
& +\left.\sum_{i=1}^{I-2 J-1} \sum_{j=0} \epsilon_{0}\left(\left(\delta_{x} E_{y}^{n+1}\right)^{2}-\left(\delta_{x} E_{y}^{n}\right)^{2}\right)\right|_{i+\frac{1}{2}, j+\frac{1}{2}} h_{i+\frac{1}{2}} k_{j+\frac{1}{2}} \\
& +\left.\sum_{i=1}^{I-1 J-1} \sum_{j=0} \epsilon_{0}\left(\left(\delta_{x} H_{z}^{n+\frac{3}{2}}\right)^{2}-\left(\delta_{x} H_{z}^{n+\Delta t}\right)^{2}\right)\right|_{i, j+\frac{1}{2}} h_{i} k_{j+\frac{1}{2}}
\end{aligned}
$$




$$
\begin{aligned}
& +\left.\sum_{i=1}^{I-1} \sum_{j=1}^{I-1}\left\{\frac{1}{\epsilon_{0} \omega_{p e}^{2}}\left(\left(\delta_{x} J_{x}^{n+\frac{3}{2}}\right)^{2}-\left(\delta_{x} J_{x}^{n+\frac{1}{2}}\right)^{2}\right)+\frac{\Delta t \Gamma_{e}}{2}\left(\delta_{x} J_{x}^{n+\frac{3}{2}}+\delta_{x} J_{x}^{n+\frac{1}{2}}\right)^{2}\right\}\right|_{i, j} h_{i} k_{j} \\
& +\left.\sum_{i=1}^{I-2 J-1}\left\{\frac{1}{\epsilon_{0} \omega_{p e}^{2}}\left(\left(\delta_{x} J_{y}^{n+\frac{3}{2}}\right)^{2}-\left(\delta_{x} J_{y}^{n+\frac{1}{2}}\right)^{2}\right)+\frac{\Delta t \Gamma_{e}}{2}\left(\delta_{x} J_{y}^{n+\frac{3}{2}}+\delta_{x} J_{y}^{n+\frac{1}{2}}\right)^{2}\right\}\right|_{i+\frac{1}{2}, j+\frac{1}{2}} h_{i+\frac{1}{2}} k_{j+\frac{1}{2}} \\
& +\left.\sum_{i=1}^{I-1} \sum_{j=0}^{J-1}\left\{\frac{1}{\mu_{0} \omega_{p m}^{2}}\left(\left(\delta_{x} K_{z}^{n+2}\right)^{2}-\left(\delta_{x} K_{z}^{n+1}\right)^{2}\right)+\frac{\Delta t \Gamma_{m}}{2}\left(\delta_{x} K_{z}^{n+2}+\delta_{x} K_{z}^{n+1}\right)^{2}\right\}\right|_{i, j+\frac{1}{2}} h_{i} k_{j+\frac{1}{2}} \\
& =\left\|\delta_{x} E_{x}^{n+1}\right\|_{\delta_{x} E_{x}}^{2}-\left\|\delta_{x} E_{x}^{n}\right\|_{\delta_{x} E_{x}}^{2}+\left\|\delta_{x} E_{y}^{n+1}\right\|_{\delta_{x} E_{y}}^{2}-\left\|\delta_{x} E_{y}^{n}\right\|_{\delta_{x} E_{y}}^{2}+\left\|\delta_{x} H_{z}^{n+\frac{3}{2}}\right\|_{\delta_{x} H_{z}}^{2}-\left\|\delta_{x} H_{z}^{n+\frac{1}{2}}\right\|_{\delta_{x} H_{z}}^{2} \\
& +\left\|\delta_{x} J_{x}^{n+\frac{3}{2}}\right\|_{\delta_{x} J_{x}}^{2}-\left\|\delta_{x} J_{x}^{n+\frac{1}{2}}\right\|_{\delta_{x} J_{x}}^{2}+\left\|\delta_{x} J_{y}^{n+\frac{3}{2}}\right\|_{\delta_{x} J_{y}}^{2}-\left\|\delta_{x} J_{y}^{n+\frac{1}{2}}\right\|_{\delta_{x} J_{y}}^{2}+\left\|\delta_{x} K_{z}^{n+2}\right\|_{\delta_{x} K_{z}}^{2}-\left\|\delta_{x} K_{z}^{n+1}\right\|_{\delta_{x} K_{z}}^{2} \\
& +\frac{\Delta t}{2}\left[\Gamma_{e}\left(\left\|\delta_{x} J_{x}^{n+\frac{3}{2}}+\delta_{x} J_{x}^{n+\frac{1}{2}}\right\|_{\delta_{x} J_{x}}^{2}+\left\|\delta_{x} J_{y}^{n+\frac{3}{2}}+\delta_{x} J_{y}^{n+\frac{1}{2}}\right\|_{\delta_{x} J_{y}}^{2}\right)+\Gamma_{m}\left\|\delta_{x} K_{z}^{n+2}+\delta_{x} K_{z}^{n+1}\right\|_{\delta_{x} K_{z}}^{2}\right] .
\end{aligned}
$$

The second equal term sign comes from the definite of mesh-dependent energy norms.

The corresponding right hand side (RHS)

$$
\begin{aligned}
\mathrm{RHS}=\Delta & \left.t \sum_{i=1}^{I-1} \sum_{j=1}^{J-1}\left(\delta_{x} \delta_{y} H_{z}^{n+\frac{1}{2}}\left(\delta_{x} E_{x}^{n+1}+\delta_{x} E_{x}^{n}\right)-\delta_{x} J_{x}^{n+\frac{1}{2}} \delta_{x} E_{x}^{n}+\delta_{x} J_{x}^{n+\frac{3}{2}} \delta_{x} E_{x}^{n+1}\right)\right|_{i, j} h_{i} k_{j} \\
& +\Delta t \sum_{i=1}^{I-2 J-1} \sum_{j=0}\left(-\delta_{x} \delta_{x} H_{z}^{n+\frac{1}{2}}\left(\delta_{x} E_{y}^{n+1}+\delta_{x} E_{y}^{n}\right)\right. \\
& \left.-\delta_{x} J_{y}^{n+\frac{1}{2}} \delta_{x} E_{y}^{n}+\delta_{x} J_{y}^{n+\frac{3}{2}} \delta_{x} E_{y}^{n+1}\right)\left.\right|_{i+\frac{1}{2}, j+\frac{1}{2}} h_{i+\frac{1}{2}} k_{j+\frac{1}{2}} \\
& +\Delta t \sum_{i=1}^{I-1} \sum_{j=0}^{J-1}\left(\left(\delta_{x} \delta_{y} E_{x}^{n+1}-\delta_{x} \delta_{x} E_{y}^{n+1}\right)\left(\delta_{x} H_{z}^{n+\frac{3}{2}}+\delta_{x} H_{z}^{n+\frac{1}{2}}\right)\right. \\
& \left.-\delta_{x} K_{z}^{n+1} \delta_{x} H_{z}^{n+\frac{1}{2}}+K_{z}^{n+2} \delta_{x} H_{z}^{n+\frac{3}{2}}\right)\left.\right|_{i, j+\frac{1}{2}} h_{i} k_{j+\frac{1}{2}} \\
=\Delta & \left.t \sum_{i=1}^{I-1} \sum_{j=1}^{J-1} \delta_{x} \delta_{y} H_{z}^{n+\frac{1}{2}} \delta_{x} E_{x}^{n}\right|_{i, j} h_{i} k_{j}+\left.\Delta t \sum_{i=1}^{I-1} \sum_{j=0}^{J-1} \delta_{x} \delta_{y} E_{x}^{n+1} \delta_{x} H_{z}^{n+\frac{3}{2}}\right|_{i, j+\frac{1}{2}} h_{i} k_{j+\frac{1}{2}} \\
& +\left.\Delta t \sum_{i=1}^{I-2 J-1} \sum_{j=0}\left(-\delta_{x} \delta_{x} H_{z}^{n+\frac{1}{2}} \delta_{x} E_{y}^{n}\right)\right|_{i+\frac{1}{2}, j+\frac{1}{2}} h_{i+\frac{1}{2}} k_{j+\frac{1}{2}} \\
& +\left.\Delta t \sum_{i=1}^{I-1} \sum_{j=0}^{J-1}\left(-\delta_{x} \delta_{x} E_{y}^{n+1} \delta_{x} H_{z}^{n+\frac{3}{2}}\right)\right|_{i, j+\frac{1}{2}} h_{i} k_{j+\frac{1}{2}} \\
& -\left.\sum_{j=0}^{J-1} E_{y}^{n} \cdot \delta_{x} H_{z}^{n+\frac{1}{2}}\right|_{1, j+\frac{1}{2}} \frac{k_{j+\frac{1}{2}}}{h_{\frac{1}{2}}}-\left.\sum_{j=0}^{J-1} E_{y}^{n} \cdot \delta_{x} H_{z}^{n+\frac{1}{2}}\right|_{I-1, j+\frac{1}{2}} \frac{k_{j+\frac{1}{2}}}{h_{I-\frac{1}{2}}} \\
&
\end{aligned}
$$




$$
\begin{aligned}
& +\left.\Delta t \sum_{i=1}^{I-1} \sum_{j=1}^{J-1}\left(\delta_{x} J_{x}^{n+\frac{1}{2}} \delta_{x} E_{x}^{n}+\delta_{x} J_{x}^{n+\frac{3}{2}} \delta_{x} E_{x}^{n+1}\right)\right|_{i, j} h_{i} k_{j} \\
& +\left.\Delta t \sum_{i=1}^{I-2 J-1} \sum_{j=0}^{n+\frac{1}{2}}\left(-\delta_{x} J_{y}^{n+\frac{1}{2}} E_{y}^{n}+\delta_{x} J_{y}^{n+\frac{3}{2}} \delta_{x} E_{y}^{n+1}\right)\right|_{i+\frac{1}{2}, j+\frac{1}{2}} h_{i+\frac{1}{2}} k_{j+\frac{1}{2}} \\
& +\left.\Delta t \sum_{i=1}^{I-1} \sum_{j=0}^{J-1}\left(-\delta_{x} K_{z}^{n+1} \delta_{x} H_{z}^{n+\frac{1}{2}}+K_{z}^{n+2} \delta_{x} H_{z}^{n+\frac{3}{2}}\right)\right|_{i, j+\frac{1}{2}} h_{i} k_{j+\frac{1}{2}} .
\end{aligned}
$$

By make use of the Lemma 3.1, the second equal term sign holds.

$$
\begin{aligned}
& \text { Summing up LHS and RHS from } n=0 \text { to } N-1 \text {, and using Lemma 3.1, we have } \\
& \begin{array}{l}
\left\|\delta_{x} E_{x}^{N}\right\|_{\delta_{x} E_{x}}^{2}-\left\|\delta_{x} E_{x}^{0}\right\|_{\delta_{x} E_{x}}^{2}+\left\|\delta_{x} E_{y}^{N}\right\|_{\delta_{x} E_{y}}^{2}-\left\|\delta_{x} E_{y}^{0}\right\|_{\delta_{x} E_{y}}^{2}+\left\|\delta_{x} H_{z}^{N+\frac{1}{2}}\right\|_{\delta_{x} H_{z}}^{2}-\left\|\delta_{x} H_{z}^{\frac{1}{2}}\right\|_{\delta_{x} H_{z}}^{2} \\
+\left\|\delta_{x} J_{x}^{N+\frac{1}{2}}\right\|_{\delta_{x} J_{x}}^{2}-\left\|\delta_{x} J_{x}^{\frac{1}{2}}\right\|_{\delta_{x} J_{x}}^{2}+\left\|\delta_{x} J_{y}^{N+\frac{1}{2}}\right\|_{\delta_{x} J_{y}}^{2}-\left\|\delta_{x} J_{y}^{\frac{1}{2}}\right\|_{\delta_{x} J_{y}}^{2}+\left\|\delta_{x} K_{z}^{N+1}\right\|_{\delta_{x} K_{z}}^{2}-\left\|\delta_{x} K_{z}^{1}\right\|_{\delta_{x} K_{z}}^{2} \\
\quad+\frac{\Delta t}{2} \sum_{n=0}^{N-1}\left[\Gamma_{e}\left(\left\|\delta_{x} J_{x}^{n+\frac{3}{2}}+\delta_{x} J_{x}^{n+\frac{1}{2}}\right\|_{\delta_{x} J_{x}}^{2}+\left\|\delta_{x} J_{y}^{n+\frac{3}{2}}+\delta_{x} J_{y}^{n+\frac{1}{2}}\right\|_{\delta_{x} J_{y}}^{2}\right)\right. \\
\left.\quad+\Gamma_{m}\left\|\delta_{x} K_{z}^{n+2}+\delta_{x} K_{z}^{n+1}\right\|_{\delta_{x} K_{z}}^{2}\right]
\end{array} \\
& =T_{1}+T_{2}+T_{3}+T_{4}
\end{aligned}
$$

where

$$
\begin{aligned}
& T_{1}=\Delta\left.t \sum_{i=1}^{I-1} \sum_{j=1}^{J-1}\left(\delta_{x} E_{x}^{0} \cdot \delta_{x} \delta_{y} H_{z}^{\frac{1}{2}}\right)\right|_{i, j} h_{i} k_{j}-\left.\Delta t \sum_{i=1}^{I-2 J-1} \sum_{j=1}\left(\delta_{x} E_{y}^{0} \cdot \delta_{x} \delta_{x} H_{z}^{\frac{1}{2}}\right)\right|_{i+\frac{1}{2}, j+\frac{1}{2}} h_{i+\frac{1}{2}} k_{j+\frac{1}{2}}, \\
& T_{2}=\Delta t\left.\sum_{i=1}^{I-1} \sum_{j=0}^{J-1}\left(\delta_{x} \delta_{y} E_{x}^{N}-\delta_{x} \delta_{x} E_{y}^{N}\right) \cdot \delta_{x} H_{z}^{N+\frac{1}{2}}\right|_{i+\frac{1}{2}, j+\frac{1}{2}} h_{i+\frac{1}{2}} k_{j+\frac{1}{2}} \\
& T_{3}=\left.\Delta t \sum_{j=0}^{J-1}\left(E_{y}^{N} \cdot \delta_{x} H_{z}^{N-\frac{1}{2}}+\sum_{n=1}^{N-1} E_{y}^{n} \cdot \delta_{x}\left(H_{z}^{n+\frac{1}{2}}+H_{z}^{n-\frac{1}{2}}\right)\right)\right|_{1, j+\frac{1}{2}} \frac{k_{j+\frac{1}{2}}}{h_{\frac{1}{2}}} \\
& \quad+\left.\Delta t \sum_{j=0}^{J-1}\left(E_{y}^{N} \cdot \delta_{x} H_{z}^{N-\frac{1}{2}}+\sum_{n=1}^{N-1} E_{y}^{n} \cdot \delta_{x}\left(H_{z}^{n+\frac{1}{2}}+H_{z}^{n-\frac{1}{2}}\right)\right)\right|_{I-1, j+\frac{1}{2}} \frac{k_{j+\frac{1}{2}}}{h_{I-\frac{1}{2}}} \\
& T_{4}=\left.\Delta t \sum_{i=1}^{I-1 J-1} \sum_{j=1}^{I-1}\left(\delta_{x} J_{x}^{N+\frac{1}{2}} \cdot \delta_{x} E_{x}^{N}-\delta_{x} J_{x}^{\frac{1}{2}} \cdot \delta_{x} E_{x}^{0}\right)\right|_{i, j} h_{i} k_{j} \\
& \quad+\left.\Delta t \sum_{i=1}^{I-2 J-1} \sum_{j=0}^{I-1}\left(\delta_{x} J_{y}^{N+\frac{1}{2}} \cdot \delta_{x} E_{y}^{N}-\delta_{x} J_{y}^{\frac{1}{2}} \cdot \delta_{x} E_{y}^{0}\right)\right|_{i+\frac{1}{2}, j+\frac{1}{2}} h_{i+\frac{1}{2}} k_{j+\frac{1}{2}} \\
& \quad+\left.\Delta t \sum_{i=1}^{I-1}\left(\delta_{j=0} K_{z}^{N+1} \cdot \delta_{x} H_{z}^{N+\frac{1}{2}}-\delta_{x} K_{z}^{1} \cdot \delta_{x} H_{z}^{\frac{1}{2}}\right)\right|_{i, j+\frac{1}{2}} h_{i} k_{j+\frac{1}{2}} .
\end{aligned}
$$


To estimate $T_{1}$, by the Cauchy-Schwartz inequality and the definition of norms $\|\cdot\|_{\delta_{x} E_{x}}$ and $\|\cdot\|_{\delta_{x} E_{y}}$, we have

$$
T_{1} \leq \frac{1}{2}\left[\left\|\delta_{x} E_{x}^{0}\right\|_{\delta_{x} E_{x}}^{2}+\left\|\delta_{x} E_{y}^{0}\right\|_{\delta_{x} E_{y}}^{2}+\left(\frac{\Delta t}{\epsilon_{0}}\right)^{2}\left(\left\|\delta_{x} \delta_{y} H_{z}^{\frac{1}{2}}\right\|_{\delta_{x} E_{x}}^{2}+\left\|\delta_{x} \delta_{x} H_{z}^{\frac{1}{2}}\right\|_{\delta_{x} E_{y}}^{2}\right)\right]
$$

Eq. (3.2c) can be rewritten as

$$
\delta_{x} H_{z}^{N+\frac{1}{2}}-\frac{\Delta t}{\mu}\left(\delta_{x} \delta_{y} E_{x}^{N}-\delta_{x} \delta_{x} E_{y}^{N}\right)=\delta_{x} H_{z}^{N-\frac{1}{2}}-\left.\frac{\Delta t}{\mu} \delta_{x} K_{z}^{N}\right|_{i, j+\frac{1}{2}}
$$

Squaring both sides of the above equation, multiplying both sides of the above equation by $\mu_{0} h_{i} k_{j+\frac{1}{2}}$.

$$
T_{2}=\frac{1}{2}\left[\left\|\delta_{x} H_{z}^{N+\frac{1}{2}}\right\|_{\delta_{x} H_{z}}^{2}+\left(\frac{\Delta t}{\mu}\right)^{2}\left\|\delta_{x} \delta_{y} E_{x}^{N}-\delta_{x} \delta_{x} E_{y}^{N}\right\|_{\delta_{x} H_{z}}^{2}-\left\|\delta_{x} H_{z}^{N-\frac{1}{2}}-\frac{\Delta t}{\mu} \delta_{x} K_{z}^{N}\right\|_{\delta_{x} H_{z}}^{2}\right] .
$$

Then, we have the following estimate

$$
T_{2} \leq \frac{1}{2}\left[\left\|\delta_{x} H_{z}^{N+\frac{1}{2}}\right\|_{\delta_{x} H_{z}}^{2}+\left(\frac{\Delta t}{\mu}\right)^{2}\left\|\delta_{x} \delta_{y} E_{x}^{N}-\delta_{x} \delta_{x} E_{y}^{N}\right\|_{\delta_{x} H_{z}}^{2}\right]
$$

To estimate $T_{3}$, take $i=i^{\prime}$, from (2.25) we can easily have

$$
\begin{aligned}
& E_{y}^{n}-\frac{\Delta t}{\epsilon_{0}} \delta_{x} H_{z}^{n+\frac{1}{2}}=E_{y}^{n+1}+\left.\frac{\Delta t}{\epsilon_{0}} J_{y}^{n+\frac{1}{2}}\right|_{i^{\prime}, j+\frac{1^{\prime}}{}} \\
& E_{y}^{n}+\frac{\Delta t}{\epsilon_{0}} \delta_{x} H_{z}^{n-\frac{1}{2}}=E_{y}^{n-1}-\left.\frac{\Delta t}{\epsilon_{0}} J_{y}^{n-\frac{1}{2}}\right|_{i^{\prime}, j+\frac{1}{2}} \\
& E_{y}^{N}+\frac{\Delta t}{\epsilon_{0}} \delta_{x} H_{z}^{N-\frac{1}{2}}=E_{y}^{N-1}-\left.\frac{\Delta t}{\epsilon_{0}} J_{y}^{N-\frac{1}{2}}\right|_{i^{\prime}, j+\frac{1}{2}}{ }^{\prime}
\end{aligned}
$$

where $i^{\prime}=1, I-1$. From these three equations and by a similar argument to that in deriving (3.6), we have

$$
\begin{aligned}
& \Delta t E_{y}^{n} \cdot \delta_{x}\left(H_{z}^{n+\frac{1}{2}}+H_{z}^{n-\frac{1}{2}}\right)=\frac{1}{2}[ \epsilon_{0}\left(E_{y}^{n-1}-\frac{\Delta t}{\epsilon_{0}} J_{y}^{n-\frac{1}{2}}\right)^{2}-\epsilon_{0}\left(E_{y}^{n+1}+\frac{\Delta t}{\epsilon_{0}} J_{y}^{n+\frac{1}{2}}\right)^{2} \\
&\left.+\frac{(\Delta t)^{2}}{\epsilon_{0}}\left(\left(\delta_{x} H_{z}^{n+\frac{1}{2}}\right)^{2}-\left(\delta_{x} H_{z}^{n-\frac{1}{2}}\right)^{2}\right)\right], \\
& \Delta t E_{y}^{N} \cdot \delta_{x} H_{z}^{N-\frac{1}{2}}=\frac{1}{2}\left[\epsilon_{0}\left(E_{y}^{N-1}-\frac{\Delta t}{\epsilon_{0}} J_{y}^{N-\frac{1}{2}}\right)^{2}-\epsilon_{0}\left(E_{y}^{N}\right)^{2}-\frac{(\Delta t)^{2}}{\epsilon_{0}}\left(\delta_{x} H_{z}^{N-\frac{1}{2}}\right)^{2}\right] .
\end{aligned}
$$


Substituting these two equations into the expression of $T_{3}$ and noting the definition of $|\cdot|_{y}$, it is found that

$$
\begin{aligned}
& T_{3}=\frac{1}{2}\left[\left|E_{y}^{0}\right|_{y}^{2}+\left|E_{y}^{1}\right|_{y}^{2}-2\left|E_{y}^{N}\right|_{y}^{2}-\frac{(\Delta t)^{2}}{\epsilon_{0}^{2}}\left|\delta_{x} H_{z}^{\frac{1}{2}}\right|_{y}^{2}+\frac{(\Delta t)^{2}}{\epsilon_{0}^{2}}\left|J_{y}^{\frac{1}{2}}\right|_{y}^{2}\right. \\
&-2 \Delta t \sum_{j=0}^{J-1} \sum_{n=1}^{N}\left(E_{y}^{n-1}+E_{y}^{n}\right) \cdot J_{y}^{n-\frac{1}{2}} \frac{k_{j+\frac{1}{2}}}{h_{\frac{1}{2}}}-2 \Delta t \sum_{j=0}^{J-1} \sum_{n=1}^{N}\left(E_{y}^{n-1}+E_{y}^{n}\right) \cdot J_{y}^{n-\frac{1}{2}} \frac{k_{j+\frac{1}{2}}}{h_{I-\frac{1}{2}}} \\
&\left.+2 \Delta t \sum_{j=0}^{J-1} E_{y}^{1} \cdot J_{y}^{\frac{1}{2}} \frac{k_{j+\frac{1}{2}}}{h_{\frac{1}{2}}}+2 \Delta t \sum_{j=0}^{J-1} E_{y}^{1} \cdot J_{y}^{\frac{1}{2}} \frac{k_{j+\frac{1}{2}}}{h_{I-\frac{1}{2}}}\right]
\end{aligned}
$$

From Eq. (2.28), we have

$$
\left(1+\frac{\Delta t \Gamma_{e}}{2}\right) J_{y}^{n+\frac{3}{2}}+\left(-1+\frac{\Delta t \Gamma_{e}}{2}\right) J_{y}^{n+\frac{1}{2}}=\left.\Delta t \epsilon_{0} \omega_{p e}^{2} E_{y}^{n+1}\right|_{i, j+\frac{1}{2}} \cdot
$$

Multiplying each side of the above equation by $E_{y}^{n+1}$, choose a constant

$$
C=\max \left\{1+\frac{\Delta t \Gamma_{e}}{2},-1+\frac{\Delta t \Gamma_{e}}{2}\right\}, \quad(C>0)
$$

Then, we can obtain that

$$
\left(J_{y}^{n+\frac{3}{2}}+J_{y}^{n+\frac{1}{2}}\right) \cdot E_{y}^{n+1} \geq\left.\frac{\Delta t \epsilon_{0} \omega_{p e}^{2}}{C}\left(E_{y}^{n+1}\right)^{2}\right|_{i, j+\frac{1}{2}} \cdot
$$

Summing up Eq. (3.10) from $n=0$ to $N-1$ and noting the definition of $|\cdot|_{y}$, we obtain

$$
\sum_{j=0}^{J-1}\left(\sum_{n=1}^{N}\left(E_{y}^{n-1}+E_{y}^{n}\right) \cdot J_{y}^{n-\frac{1}{2}}+E_{y}^{N} \cdot J_{y}^{N+\frac{1}{2}}-E_{y}^{0} \cdot J_{y}^{\frac{1}{2}}\right) \frac{k_{j+\frac{1}{2}}}{h_{\frac{1}{2}}} \geq \frac{\Delta t \omega_{p e}^{2}}{C} \sum_{n=0}^{N-1}\left|E_{y}^{n+1}\right|_{y}^{2} .
$$

Combining Eq. (3.11) and (3.9), by the Cauchy-Schwartz inequality, it follows that

$$
\begin{aligned}
T_{3} \leq & \frac{1}{2}\left[2\left|E_{y}^{0}\right|_{y}^{2}+2\left|E_{y}^{1}\right|_{y}^{2}-2\left|E_{y}^{N}\right|_{y}^{2}+3\left(\frac{\Delta t}{\epsilon_{0}}\right)^{2}\left|J_{y}^{\frac{1}{2}}\right|_{y}^{2}\right. \\
& \left.+2 \Delta t \sum_{j=0}^{J-1} E_{y}^{N} \cdot J_{y}^{N+\frac{1}{2}} \frac{k_{j+\frac{1}{2}}}{h_{\frac{1}{2}}}+2 \Delta t \sum_{j=0}^{J-1} E_{y}^{N} \cdot J_{y}^{N+\frac{1}{2}} \frac{k_{j+\frac{1}{2}}}{h_{I-\frac{1}{2}}}\right]
\end{aligned}
$$


By Remark (2.32), we known that

$$
\begin{aligned}
& 2 \Delta t \sum_{j=0}^{J-1} E_{y}^{N} J_{y}^{N+\frac{1}{2}} \frac{k_{j+\frac{1}{2}}}{h_{\frac{1}{2}}} \leq \sum_{j=0}^{J-1} \epsilon_{0}\left(E_{y}^{N}\right)^{2} \frac{k_{j+\frac{1}{2}}}{h_{\frac{1}{2}}}+\frac{\Delta t}{\epsilon_{0}} \sum_{j=0}^{J-1}\left(J_{y}^{N+\frac{1}{2}}-0\right)^{2} \frac{k_{j+\frac{1}{2}}}{h_{\frac{1}{2}}} \\
& =\sum_{j=0}^{J-1} \epsilon_{0}\left(E_{y}^{N}\right)^{2} \frac{k_{j+\frac{1}{2}}}{h_{\frac{1}{2}}}+\frac{\Delta t}{\epsilon_{0}} \sum_{j=0}^{J-1}\left(\delta_{x} J_{y}^{N+\frac{1}{2}}\right)^{2} k_{j+\frac{1}{2}} h_{\frac{1}{2}} \\
& 2 \Delta t \sum_{j=0}^{J-1} E_{y}^{N} J_{y}^{N+\frac{1}{2}} \frac{k_{j+\frac{1}{2}}}{h_{I-\frac{1}{2}}} \leq \sum_{j=0}^{J-1} \epsilon_{0}\left(E_{y}^{N}\right)^{2} \frac{k_{j+\frac{1}{2}}}{h_{I-\frac{1}{2}}}+\frac{\Delta t}{\epsilon_{0}} \sum_{j=0}^{J-1}\left(\delta_{x} J_{y}^{N+\frac{1}{2}}\right)^{2} k_{j+\frac{1}{2}} h_{I-\frac{1}{2}} .
\end{aligned}
$$

From above equation, we obtain that

$$
T_{3} \leq \frac{1}{2}\left[2\left|E_{y}^{0}\right|_{y}^{2}+2\left|E_{y}^{1}\right|_{y}^{2}-\left|E_{y}^{N}\right|_{y}^{2}+3\left(\frac{\Delta t}{\epsilon_{0}}\right)^{2}\left|J_{y}^{\frac{1}{2}}\right|_{y}^{2}+\left(\Delta t \omega_{p e}\right)^{2}\left\|\delta_{x} J_{y}^{N+\frac{1}{2}}\right\|_{\delta_{x} J_{y}}^{2}\right] .
$$

To estimate $T_{4}$, using the Cauchy-Schwartz inequality, it follows that

$$
\begin{aligned}
T_{4} \leq \frac{1}{4} \| & \delta_{x} E_{x}^{N}\left\|_{\delta_{x} E_{x}}^{2}+\left(\Delta t \omega_{p e}\right)^{2}\right\| \delta_{x} J_{x}^{N+\frac{1}{2}}\left\|_{\delta_{x} J_{x}}^{2}+\frac{1}{4}\right\| \delta_{x} E_{x}^{0}\left\|_{\delta_{x} E_{x}}^{2}+\left(\Delta t \omega_{p e}\right)^{2}\right\| \delta_{x} J_{x}^{\frac{1}{2}} \|_{\delta_{x} J_{x}}^{2} \\
& +\frac{1}{4}\left\|\delta_{x} E_{y}^{N}\right\|_{\delta_{x} E_{y}}^{2}+\left(\Delta t \omega_{p e}\right)^{2}\left\|\delta_{x} J_{y}^{N+\frac{1}{2}}\right\|_{\delta_{x} J_{y}}^{2}+\frac{1}{4}\left\|\delta_{x} E_{y}^{0}\right\|_{\delta_{x} E_{y}}^{2}+\left(\Delta t \omega_{p e}\right)^{2}\left\|\delta_{x} J_{y}^{\frac{1}{2}}\right\|_{\delta_{x} J_{y}}^{2} \\
& +\frac{1}{4}\left\|\delta_{x} H_{z}^{N+\frac{1}{2}}\right\|_{\delta_{x} H_{z}}^{2}+\left(\Delta t \omega_{p m}\right)^{2}\left\|\delta_{x} K_{z}^{N+1}\right\|_{\delta_{x} K_{z}}^{2} \\
& +\frac{1}{4}\left\|\delta_{x} H_{z}^{\frac{1}{2}}\right\|_{\delta_{x} H_{z}}^{2}+\left(\Delta t \omega_{p m}\right)^{2}\left\|\delta_{x} K_{z}^{1}\right\|_{\delta_{x} K_{z}}^{2}
\end{aligned}
$$

Combining (3.4), (3.6), (3.12), (3.13) with (3.3) gives

$$
\begin{aligned}
& \frac{3}{4}\left(\left\|\delta_{x} E_{x}^{N}\right\|_{\delta_{x} E_{x}}^{2}+\left\|\delta_{x} E_{y}^{N}\right\|_{\delta_{x} E_{y}}^{2}\right)+\frac{1}{4}\left\|\delta_{x} H_{z}^{N+\frac{1}{2}}\right\|_{\delta_{x} H_{z}}^{2} \\
& \quad+\left(1-\left(\Delta t \omega_{p e}\right)^{2}\right)\left(\left\|\delta_{x} J_{x}^{N+\frac{1}{2}}\right\|_{\delta_{x} J_{x}}^{2}+\left\|\delta_{x} J_{y}^{N+\frac{1}{2}}\right\|_{\delta_{x} J_{y}}^{2}\right)+\left(1-\left(\Delta t \omega_{p m}\right)^{2}\right)\left\|\delta_{x} K_{z}^{N+1}\right\|_{\delta_{x} K_{z}}^{2} \\
& \leq \frac{7}{4}\left(\left\|\delta_{x} E_{x}^{0}\right\|_{\delta_{x} E_{x}}^{2}+\left\|\delta_{x} E_{y}^{0}\right\|_{\delta_{x} E_{y}}^{2}\right)+\frac{5}{4}\left\|\delta_{x} H_{z}^{\frac{1}{2}}\right\|_{\delta_{x} H_{z}}^{2} \\
& \quad+\left(1+\left(\Delta t \omega_{p e}\right)^{2}\right)\left(\left\|\delta_{x} J_{x}^{\frac{1}{2}}\right\|_{\delta_{x} J_{x}}^{2}+\left\|\delta_{x} J_{y}^{\frac{1}{2}}\right\|_{\delta_{x} J_{y}}^{2}\right)+\left(1+\left(\Delta t \omega_{p m}\right)^{2}\right)\left\|\delta_{x} K_{z}^{1}\right\|_{\delta_{x} K_{z}}^{2} \\
& \quad+G_{1}+G_{2}
\end{aligned}
$$

where

$$
\begin{aligned}
& G_{1}=\left(\frac{\Delta t}{\sqrt{2} \epsilon_{0}}\right)^{2}\left[\left\|\delta_{x} \delta_{y} H_{z}^{\frac{1}{2}}\right\|_{\delta_{x} E_{x}}^{2}+\left\|\delta_{x} \delta_{x} H_{z}^{\frac{1}{2}}\right\|_{\delta_{x} E_{y}}^{2}\right]+\left(\frac{\Delta t}{\sqrt{2} \mu}\right)^{2}\left\|\delta_{x} \delta_{y} E_{x}^{N}-\delta_{x} \delta_{x} E_{y}^{N}\right\|_{\delta_{x} H_{z^{\prime}}}^{2} \\
& G_{2}=\frac{1}{2}\left[2\left|E_{y}^{0}\right|_{y}^{2}+2\left|E_{y}^{1}\right|_{y}^{2}-\left|E_{y}^{N}\right|_{y}^{2}+3\left(\frac{\Delta t}{\epsilon_{0}}\right)^{2}\left|J_{y}^{\frac{1}{2}}\right|_{y}^{2}+\left(\Delta t \omega_{p e}\right)^{2}\left\|\delta_{x} J_{y}^{N+\frac{1}{2}}\right\|_{\delta_{x} J_{y}}^{2}\right]
\end{aligned}
$$


Similarly, we obtain, by replacing $\delta_{x}$ with $\delta_{y}$, that

$$
\begin{aligned}
& \frac{3}{4}\left(\left\|\delta_{y} E_{x}^{N}\right\|_{\delta_{y} E_{x}}^{2}+\left\|\delta_{y} E_{y}^{N}\right\|_{\delta_{y} E_{y}}^{2}\right)+\frac{1}{4}\left\|\delta_{y} H_{z}^{N+\frac{1}{2}}\right\|_{\delta_{y} H_{z}}^{2} \\
& +\left(1-\left(\Delta t \omega_{p e}\right)^{2}\right)\left(\left\|\delta_{y} J_{x}^{N+\frac{1}{2}}\right\|_{\delta_{y} J_{x}}^{2}+\left\|\delta_{y} J_{y}^{N+\frac{1}{2}}\right\|_{\delta_{y} J_{y}}^{2}\right)+\left(1-\left(\Delta t \omega_{p m}\right)^{2}\right)\left\|\delta_{y} K_{z}^{N+1}\right\|_{\delta_{y} K_{z}}^{2} \\
\leq & \frac{7}{4}\left(\left\|\delta_{y} E_{x}^{0}\right\|_{\delta_{y} E_{x}}^{2}+\left\|\delta_{y} E_{y}^{0}\right\|_{\delta_{y} E_{y}}^{2}\right)+\frac{5}{4}\left\|\delta_{y} H_{z}^{\frac{1}{2}}\right\|_{\delta_{y} H_{z}}^{2} \\
& +\left(1+\left(\Delta t \omega_{p e}\right)^{2}\right)\left(\left\|\delta_{y} J_{x}^{\frac{1}{2}}\right\|_{\delta_{y} J_{x}}^{2}+\left\|\delta_{y} J_{y}^{\frac{1}{2}}\right\|_{\delta_{y} J_{y}}^{2}\right)+\left(1+\left(\Delta t \omega_{p m}\right)^{2}\right)\left\|\delta_{y} K_{z}^{1}\right\|_{\delta_{y} K_{z}}^{2} \\
& +\hat{G}_{1}+\hat{G}_{2}
\end{aligned}
$$

where

$$
\begin{aligned}
& \hat{G}_{1}=\left(\frac{\Delta t}{\sqrt{2} \epsilon_{0}}\right)^{2}\left[\left\|\delta_{y} \delta_{y} H_{z}^{\frac{1}{2}}\right\|_{\delta_{y} E_{x}}^{2}+\left\|\delta_{y} \delta_{x} H_{z}^{\frac{1}{2}}\right\|_{\delta_{y} E_{y}}^{2}\right]+\left(\frac{\Delta t}{\sqrt{2} \mu}\right)^{2}\left\|\delta_{y} \delta_{y} E_{x}^{N}-\delta_{y} \delta_{x} E_{y}^{N}\right\|_{\delta_{y} H_{z}^{\prime}}^{2} \\
& \hat{G}_{2}=\frac{1}{2}\left[2\left|E_{x}^{0}\right|_{x}^{2}+2\left|E_{x}^{1}\right|_{x}^{2}-\left|E_{x}^{N}\right|_{x}^{2}+3\left(\frac{\Delta t}{\epsilon_{0}}\right)^{2}\left|J_{x}^{\frac{1}{2}}\right|_{x}^{2}+\left(\Delta t \omega_{p e}\right)^{2}\left\|\delta_{y} J_{x}^{N+\frac{1}{2}}\right\|_{\delta_{y} J_{x}}^{2}\right] .
\end{aligned}
$$

For the first term of $G_{1}$ and $\hat{G}_{1}$, by the definition of norms $\|\cdot\|_{\delta_{x} E_{x}}$ and $\|\cdot\|_{\delta_{y} H_{z}}$, using the Cauchy-Schwartz inequality, it can be shown that

$$
\begin{aligned}
& \left\|\delta_{x} \delta_{y} H_{z}^{\frac{1}{2}}\right\|_{\delta_{x} E_{x}}^{2}=\sum_{i=1}^{I-1} \sum_{j=1}^{J-1} \epsilon_{0}\left[\frac{\delta_{y} H_{z_{i+\frac{1}{2}, j}^{\frac{1}{2}}}-\delta_{y} H_{z_{i-\frac{1}{2}, j}^{\frac{1}{2}}}}{h_{i}}\right]^{2} h_{i} k_{j} \\
& \leq \sum_{i=1}^{I-1} \sum_{j=1}^{J-1} \epsilon_{0}\left[\frac{2}{h_{i} h_{i+\frac{1}{2}}}\left(\delta_{y} H_{z_{i+\frac{1}{2}, j}^{\frac{1}{2}}}\right)^{2} h_{i+\frac{1}{2}} k_{j}+\frac{2}{h_{i} h_{i-\frac{1}{2}}}\left(\delta_{y} H_{z_{i-\frac{1}{2}, j}}^{\frac{1}{2}}\right)^{2} h_{i-\frac{1}{2}} k_{j}\right] \\
& \leq \frac{4 \epsilon_{0}}{\mu_{0} h_{\min }^{2}}\left\|\delta_{y} H_{z}^{\frac{1}{2}}\right\|_{\delta_{y} H_{z^{\prime}}}^{2} \\
& \left\|\delta_{x} \delta_{x} H_{z}^{\frac{1}{2}}\right\|_{\delta_{x} E_{y}}^{2} \leq \frac{4 \epsilon_{0}}{\mu_{0} h_{\min }^{2}}\left\|\delta_{x} H_{z}^{\frac{1}{2}}\right\|_{\delta_{x} H_{z^{\prime}}}^{2} \\
& \left\|\delta_{y} \delta_{x} H_{z}^{\frac{1}{2}}\right\|_{\delta_{y} E_{y}}^{2} \leq \frac{4 \epsilon_{0}}{\mu_{0} k_{\min }^{2}}\left\|\delta_{x} H_{z}^{\frac{1}{2}}\right\|_{\delta_{x} H_{z^{\prime}}}^{2} \\
& \left\|\delta_{y} \delta_{y} H_{z}^{\frac{1}{2}}\right\|_{\delta_{y} E_{x}}^{2} \leq \frac{4 \epsilon_{0}}{\mu_{0} k_{\min }^{2}}\left\|\delta_{y} H_{z}^{\frac{1}{2}}\right\|_{\delta_{y} H_{z}}^{2} .
\end{aligned}
$$

For the second term of $G_{1}$ and $\hat{G}_{1}$.

$$
\left\|\delta_{x}\left(\delta_{y} E_{x}^{N}-\delta_{x} E_{y}^{N}\right)\right\|_{\delta_{x} H_{z}}^{2}
$$




$$
\begin{aligned}
& =\sum_{i=1}^{I-1} \sum_{j=0}^{J-1} \mu_{0}\left(\delta_{x} \delta_{y} E_{x}^{N}\right)^{2} h_{i} k_{j+\frac{1}{2}}+\sum_{i=1}^{I-1} \sum_{j=0}^{J-1} \mu_{0}\left(\delta_{x} \delta_{x} E_{y}^{N}\right)^{2} h_{i} k_{j+\frac{1}{2}} \\
& \quad-2 \sum_{i=1}^{I-1} \sum_{j=0}^{J-1} \mu_{0} \delta_{x} \delta_{y} E_{x}^{N} \cdot \delta_{x} \delta_{x} E_{y}^{N} h_{i} k_{j+\frac{1}{2}} \\
& =: A_{1}+A_{2}+A_{3} .
\end{aligned}
$$

By the Cauchy-Schwartz inequality and the definition of the norms $\|\cdot\|_{\delta_{y} E_{x}},\left.\|\cdot\|_{\delta_{x} E_{y}}|\cdot|\right|_{x}$ and $|\cdot| y$, we have

$$
\begin{aligned}
& A_{1}=\sum_{i=1}^{I-1} \sum_{j=0}^{J-1} \mu_{0}\left[\frac{\delta_{y} E_{x_{i+\frac{1}{2}, j+\frac{1}{2}}^{N}}-\delta_{y} E_{x_{i-\frac{1}{2}, j+\frac{1}{2}}}^{N}}{h_{i}}\right]^{2} h_{i} k_{j+\frac{1}{2}} \\
& \leq \sum_{i=1}^{I-1} \sum_{j=0}^{J-1} \mu_{0}\left[\frac{2}{h_{i} h_{i+\frac{1}{2}}}\left(\delta_{y} E_{x_{i+\frac{1}{2}, j+\frac{1}{2}}^{N}}\right)^{2} h_{i+\frac{1}{2}} k_{j+\frac{1}{2}}+\frac{2}{h_{i} h_{i-\frac{1}{2}}}\left(\delta_{y} E_{x_{i-\frac{1}{2}, j+\frac{1}{2}}^{N}}\right)^{2} h_{i-\frac{1}{2}} k_{j+\frac{1}{2}}\right] \\
& \leq \frac{4 \mu_{0}}{\epsilon_{0} h_{\min }^{2}}\left(\left\|\delta_{y} E_{x}^{N}\right\|_{\delta_{y} E_{x}}^{2}+\left|E_{x}^{N}\right|_{x}^{2}\right) \\
& A_{2} \leq \frac{4 \mu_{0}}{\epsilon_{0} h_{\min }^{2}}\left(\left\|\delta_{x} E_{y}^{N}\right\|_{\delta_{x} E_{y}}^{2}+\left|E_{y}^{N}\right|_{y}^{2}\right) .
\end{aligned}
$$

To estimate $A_{3}$, we have

$$
\begin{aligned}
A_{3}= & 2 \sum_{i=1}^{I-1} \sum_{j=1}^{J-1} \mu_{0} \delta_{x} E_{x}^{N} \cdot \delta_{x} \delta_{x} \delta_{y} E_{y}^{N} h_{i} k_{j} \\
= & 2 \sum_{i=1}^{I-1} \sum_{j=0}^{J-1} \mu_{0} \delta_{x} E_{x}^{N} \cdot \delta_{x} \delta_{x} \delta_{y} E_{y}^{N} h_{i} k_{j} \\
= & -2 \sum_{i=1}^{I-2} \sum_{j=1}^{J-1} \mu_{0} \delta_{x} \delta_{x} E_{x_{i+\frac{1}{2}, j}}^{N} \cdot \delta_{x} \delta_{y} E_{y_{i+\frac{1}{2}, j}}^{N} h_{i+\frac{1}{2}} k_{j} \\
& \quad+2 \mu_{0} \sum_{j=1}^{J-1}\left[\delta_{x} E_{x_{1, j}}^{N} \cdot \delta_{x} \delta_{y} E_{y_{\frac{1}{2}, j}}^{N} \frac{k_{j}}{h_{\frac{1}{2}}}-\delta_{x} E_{x_{I-1, j}}^{N} \cdot \delta_{x} \delta_{y} E_{y_{I-\frac{1}{2}, j}}^{N} \frac{k_{j}}{h_{I-\frac{1}{2}}}\right] .
\end{aligned}
$$

The first and third equal sign come from Lemma 3.1, the second equal sign comes from the PEC boundary condition (2.30).

Using the similar technique as in (3.19a), and the PEC boundary condition (2.30), we have

$$
A_{3} \leq \frac{4 \mu_{0}}{\epsilon_{0} h_{\min }^{2}}\left(\left\|\delta_{x} E_{x}^{N}\right\|_{\delta_{x} E_{x}}^{2}+\left\|\delta_{y} E_{y}^{N}\right\|_{\delta_{y} E_{y}}^{2}\right)
$$


Combining the (3.19a), (3.19b) and (3.20) give

$$
\begin{aligned}
& \left\|\delta_{x}\left(\delta_{y} E_{x}^{N}-\delta_{x} E_{y}^{N}\right)\right\|_{\delta_{x} H_{z}}^{2} \\
& \leq \frac{4 \mu}{\epsilon_{0} h_{\min }^{2}}\left(\left\|\delta_{y} E_{x}^{N}\right\|_{\delta_{y} E_{x}}^{2}+\left|E_{x}^{N}\right|_{x}^{2}+\left\|\delta_{x} E_{y}^{N}\right\|_{\delta_{x} E_{y}}^{2}\right. \\
& \left.\quad+\left|E_{y}^{N}\right|_{y}^{2}+\left\|\delta_{x} E_{x}^{N}\right\|_{\delta_{x} E_{x}}^{2}+\left\|\delta_{y} E_{y}^{N}\right\|_{\delta_{y} E_{y}}^{2}\right) .
\end{aligned}
$$

Similarly, we have

$$
\begin{gathered}
\left\|\delta_{y}\left(\delta_{y} E_{x}^{N}-\delta_{x} E_{y}^{N}\right)\right\|_{\delta_{y} H_{z}}^{2} \\
\leq \frac{4 \mu}{\epsilon_{0} k_{\min }^{2}}\left(\left\|\delta_{y} E_{x}^{N}\right\|_{\delta_{y} E_{x}}^{2}+\left|E_{x}^{N}\right|_{x}^{2}+\left\|\delta_{x} E_{y}^{N}\right\|_{\delta_{x} E_{y}}^{2}\right. \\
\left.\quad+\left|E_{y}^{N}\right|_{y}^{2}+\left\|\delta_{x} E_{x}^{N}\right\|_{\delta_{x} E_{x}}^{2}+\left\|\delta_{y} E_{y}^{N}\right\|_{\delta_{y} E_{y}}^{2}\right) .
\end{gathered}
$$

Combining (3.18a)-(3.18d), (3.21), (3.22) with (3.15a) and (3.17a), we have

$$
\begin{aligned}
G_{1}+\hat{G}_{1} \leq & \frac{2(\Delta t)^{2}}{\mu_{0} \epsilon_{0}}\left(\frac{1}{h_{\min }^{2}}+\frac{1}{k_{\min }^{2}}\right)\left(\left\|\delta_{x} H_{z}^{\frac{1}{2}}\right\|_{\delta_{x} H_{z}}^{2}+\left\|\delta_{y} H_{z}^{\frac{1}{2}}\right\|_{\delta_{y} H_{z}}^{2}\right. \\
& +\left\|\delta_{y} E_{x}^{N}\right\|_{\delta_{y} E_{x}}^{2}+\left|E_{x}^{N}\right|_{x}^{2}+\left\|\delta_{x} E_{y}^{N}\right\|_{\delta_{x} E_{y}}^{2} \\
& \left.+\left|E_{y}^{N}\right|_{y}^{2}+\left\|\delta_{x} E_{x}^{N}\right\|_{\delta_{x} E_{x}}^{2}+\left\|\delta_{y} E_{y}^{N}\right\|_{\delta_{y} E_{y}}^{2}\right) .
\end{aligned}
$$

The key of estimate $G_{2}$ and $\hat{G}_{2}$ is to eliminate $E_{x}^{1}$ and $E_{y}^{1}$. Take $j=j^{\prime}$ in (2.24), $i=i^{\prime}$ in (2.25) and let $m=0$ in both equations to get

$$
\begin{aligned}
& E_{x}^{1}=E_{x}^{0}+\frac{\Delta t}{\epsilon_{0}} \delta_{y} H_{z}^{\frac{1}{2}}-\left.\frac{\Delta t}{\epsilon_{0}} J_{x}^{\frac{1}{2}}\right|_{i+\frac{1}{2}, j^{\prime}}, \\
& E_{y}^{1}=E_{y}^{0}-\frac{\Delta t}{\epsilon_{0}} \delta_{x} H_{z}^{\frac{1}{2}}-\left.\frac{\Delta t}{\epsilon_{0}} J_{y}^{\frac{1}{2}}\right|_{i^{\prime}, j+\frac{1}{2}} .
\end{aligned}
$$

where $i^{\prime}=1, I-1$ and $j^{\prime}=1, J-1$. Thus, together with the definitions of $|\cdot|_{x}$ and $|\cdot|_{y}$ implies that

$$
\begin{aligned}
& \left|E_{x}^{1}\right|_{x}^{2} \leq 2\left|E_{x}^{0}\right|_{x}^{2}+4\left(\frac{\Delta t}{\epsilon_{0}}\right)^{2}\left|\delta_{y} H_{z}^{\frac{1}{2}}\right|_{x}^{2}+4\left(\frac{\Delta t}{\epsilon_{0}}\right)^{2}\left|J_{x}^{\frac{1}{2}}\right|_{x}^{2} \\
& \left|E_{y}^{1}\right|_{y}^{2} \leq 2\left|E_{y}^{0}\right|_{y}^{2}+4\left(\frac{\Delta t}{\epsilon_{0}}\right)^{2}\left|\delta_{x} H_{z}^{\frac{1}{2}}\right|_{y}^{2}+4\left(\frac{\Delta t}{\epsilon_{0}}\right)^{2}\left|J_{y}^{\frac{1}{2}}\right|_{y}^{2} .
\end{aligned}
$$


Then, we have

$$
\begin{aligned}
G_{2}+\hat{G}_{2} \leq & \frac{1}{2}[ \\
& 6\left(\left|E_{x}^{0}\right|_{x}^{2}+\left|E_{y}^{0}\right|_{y}^{2}\right)-\left|E_{x}^{N}\right|_{x}^{2}-\left|E_{y}^{N}\right|_{y}^{2}+11\left(\frac{\Delta t}{\epsilon_{0}}\right)^{2}\left(\left|J_{y}^{\frac{1}{2}}\right|_{y}^{2}+\left|J_{x}^{\frac{1}{2}}\right|_{x}^{2}\right) \\
& +\left(\Delta t \omega_{p e}\right)^{2}\left(\left\|\delta_{y} J_{x}^{N+\frac{1}{2}}\right\|_{\delta_{y} J_{x}}^{2}+\left\|\delta_{x} J_{y}^{N+\frac{1}{2}}\right\|_{\delta_{x} J_{y}}^{2}\right) \\
& \left.+8\left(\frac{\Delta t}{\epsilon_{0}}\right)^{2}\left(\left|\delta_{x} H_{z}^{\frac{1}{2}}\right|_{y}^{2}+\left|\delta_{y} H_{z}^{\frac{1}{2}}\right|_{x}^{2}\right)\right]
\end{aligned}
$$

\section{Denote}

$$
C_{c f l}=\frac{(\Delta t)^{2}}{\mu_{0} \epsilon_{0}}\left(\frac{1}{h_{\min }^{2}}+\frac{1}{k_{\min }^{2}}\right),
$$

combining (3.14), (3.16), (3.23) and (3.24) gives

$$
\begin{aligned}
\left(\frac{3}{4}\right. & \left.-2 C_{c f l}\right)\left(\left\|\delta_{x} E_{x}^{N}\right\|_{\delta_{x} E_{x}}^{2}+\left\|\delta_{x} E_{y}^{N}\right\|_{\delta_{x} E_{y}}^{2}+\left\|\delta_{y} E_{x}^{N}\right\|_{\delta_{y} E_{x}}^{2}+\left\|\delta_{y} E_{y}^{N}\right\|_{\delta_{y} E_{y}}^{2}\right) \\
& +\left(\frac{1}{2}-2 C_{c f l}\right)\left(\left|E_{x}^{N}\right|_{x}^{2}+\left|E_{y}^{N}\right|_{y}^{2}\right)+\frac{1}{4}\left(\left\|\delta_{x} H_{z}^{N+\frac{1}{2}}\right\|_{\delta_{x} H_{z}}^{2}+\left\|\delta_{y} H_{z}^{N+\frac{1}{2}}\right\|_{\delta_{y} H_{z}}^{2}\right) \\
& +\left(1-\left(\Delta t \omega_{p e}\right)^{2}\right)\left(\left\|\delta_{x} J_{x}^{N+\frac{1}{2}}\right\|_{\delta_{x} J_{x}}^{2}+\left\|\delta_{y} J_{y}^{N+\frac{1}{2}}\right\|_{\delta_{y} J_{y}}^{2}\right) \\
& +\left(1-\frac{3}{2}\left(\Delta t \omega_{p e}\right)^{2}\right)\left(\left\|\delta_{x} J_{y}^{N+\frac{1}{2}}\right\|_{\delta_{x} J_{y}}^{2}+\left\|\delta_{y} J_{x}^{N+\frac{1}{2}}\right\|_{\delta_{y} J_{x}}^{2}\right) \\
& +\left(1-\left(\Delta t \omega_{p m}\right)^{2}\right)\left(\left\|\delta_{x} K_{z}^{N+1}\right\|_{\delta_{x} K_{z}}^{2}+\left\|\delta_{y} K_{z}^{N+1}\right\|_{\delta_{y} K_{z}}^{2}\right) \\
\leq & \frac{7}{4} \\
& \left.\left\|\delta_{x} E_{x}^{0}\right\|_{\delta_{x} E_{x}}^{2}+\left\|\delta_{x} E_{y}^{0}\right\|_{\delta_{x} E_{y}}^{2}+\left\|\delta_{y} E_{x}^{0}\right\|_{\delta_{y} E_{x}}^{2}+\left\|\delta_{y} E_{y}^{0}\right\|_{\delta_{y} E_{y}}^{2}\right) \\
& +3\left(\left|E_{x}^{0}\right|_{x}^{2}+\left|E_{y}^{0}\right|_{y}^{2}\right)+\left(\frac{5}{4}+2 C_{c f l}\right)\left(\left\|\delta_{x} H_{z}^{\frac{1}{2}}\right\|_{\delta_{x} H_{z}}^{2}+\left\|\delta_{y} H_{z}^{\frac{1}{2}}\right\|_{\delta_{y} H_{z}}^{2}\right) \\
& +\left(1+\left(\Delta t \omega_{p e}\right)^{2}\right)\left(\left\|\delta_{x} J_{x}^{\frac{1}{2}}\right\|_{\delta_{x} J_{x}}^{2}+\left\|\delta_{x} J_{y}^{\frac{1}{2}}\right\|_{\delta_{x} J_{y}}^{2}+\left\|\delta_{y} J_{x}^{\frac{1}{2}}\right\|_{\delta_{y} J_{x}}^{2}+\left\|\delta_{y} J_{y}^{\frac{1}{2}}\right\|_{\delta_{y} J_{y}}^{2}\right) \\
& +\left(1+\left(\Delta t \omega_{p m}\right)^{2}\right)\left(\left\|\delta_{x} K_{z}^{1}\right\|_{\delta_{x} K_{z}}^{2}+\left\|\delta_{y} K_{z}^{1}\right\|_{\delta_{y} K_{z}}^{2}\right) \\
& +\frac{11(\Delta t)^{2}}{2 \epsilon_{0}^{2}}\left(\left|J_{x}^{\frac{1}{2}}\right|_{x}^{2}+\left|J_{y}^{\frac{1}{2}}\right|_{y}^{2}\right)+\frac{4(\Delta t)^{2}}{\epsilon_{0}^{2}}\left(\left|\delta_{x} H_{z}^{\frac{1}{2}}\right|_{y}^{2}+\left|\delta_{y} H_{z}^{\frac{1}{2}}\right|_{x}^{2}\right) \\
& +\left(1+\left(\Delta t \omega_{p e}\right)^{2}\right)\left(\left\|\delta_{x} K_{z}^{1}\right\|_{\delta_{x} K_{z}}^{2}+\left\|\delta_{y} K_{z}^{1}\right\|_{\delta_{y} K_{z}}^{2}\right)
\end{aligned}
$$

which implies the required estimate (3.1) if

$$
\Delta t<\min \left\{\frac{\sqrt{\mu_{0} \epsilon_{0}}}{2} \sqrt{\frac{h_{\min }^{2} k_{\min }^{2}}{h_{\min }^{2}+k_{\min }^{2}}}, \frac{\sqrt{2}}{\sqrt{3} \omega_{p e}}, \frac{1}{\omega_{p m}}\right\} .
$$


We can conclude the proof of the Theorem 3.1.

\subsection{Stability of the Yee scheme in the discrete $L^{2}$ and $H^{1}$ norm}

Replacing $\delta_{x}$ by the identity operator $I$ and arguing similarly to that in the proof of Theorem 3.2. We can prove the following result.

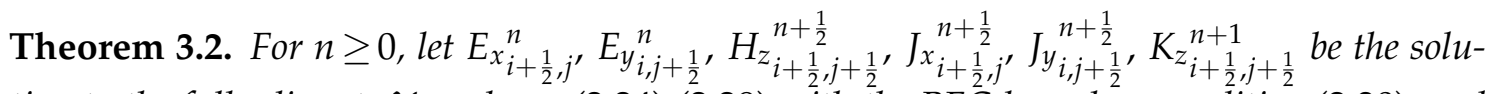
tion to the fully discrete Yee scheme (2.24)-(2.29) with the PEC boundary condition (2.30), and possesses the following regularity property:

$$
\begin{aligned}
& E_{x}, E_{y}, H_{z} \in C^{1}\left([0, T], C^{2}(\bar{\Omega})\right), \\
& J_{x}, J_{y}, K_{z} \in C^{1}\left([0, T], C^{1}(\bar{\Omega})\right) .
\end{aligned}
$$

If the time step size constraint

$$
\Delta t<\min \left\{\frac{\sqrt{\mu_{0} \epsilon_{0}}}{2} \sqrt{\frac{h_{\min }^{2} k_{\min }^{2}}{h_{\min }^{2}+k_{\min }^{2}}}, \frac{\sqrt{2}}{\sqrt{3} \omega_{p e}}, \frac{1}{\omega_{p m}}\right\}
$$

is satisfied, then the following estimate holds:

$$
\begin{aligned}
& \left\|E_{x}^{n}\right\|_{E_{x}}^{2}+\left\|E_{y}^{n}\right\|_{E_{y}}^{2}+\left\|J_{x}^{n+\frac{1}{2}}\right\|_{J_{x}}^{2}+\left\|J_{y}^{n+\frac{1}{2}}\right\|_{J_{y}}^{2}+\left\|H_{z}^{n+\frac{1}{2}}\right\|_{H_{z}}^{2}+\left\|K_{z}^{n+1}\right\|_{K_{z}}^{2} \\
\leq & C\left\{\left\|E_{x}^{0}\right\|_{E_{x}}^{2}+\left\|E_{y}^{0}\right\|_{E_{y}}^{2}+\left\|J_{x}^{\frac{1}{2}}\right\|_{J_{x}}^{2}+\left\|J_{y}^{\frac{1}{2}}\right\|_{J_{y}}^{2}+\left\|H_{z}^{\frac{1}{2}}\right\|_{H_{z}}^{2}+\left\|K_{z}^{1}\right\|_{K_{z}}^{2}\right\} .
\end{aligned}
$$

Combining Theorems 3.1 and Theorems 3.2, we can obtain the stability result in the discrete $H^{1}$ norm.

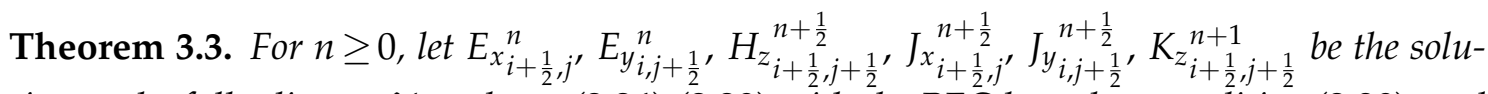
tion to the fully discrete Yee scheme (2.24)-(2.29) with the PEC boundary condition (2.30), and possesses the following regularity property:

$$
\begin{aligned}
& E_{x}, E_{y}, H_{z} \in C^{1}\left([0, T], C^{2}(\bar{\Omega})\right), \\
& J_{x}, J_{y}, K_{z} \in C^{1}\left([0, T], C^{1}(\bar{\Omega})\right) .
\end{aligned}
$$

If the time step size constraint

$$
\Delta t<\min \left\{\frac{\sqrt{\mu_{0} \epsilon_{0}}}{2} \sqrt{\frac{h_{\min }^{2} k_{\min }^{2}}{h_{\min }^{2}+k_{\min }^{2}}}, \frac{\sqrt{2}}{\sqrt{3} \omega_{p e}}, \frac{1}{\omega_{p m}}\right\}
$$

is satisfied, then the following estimate holds:

$$
\left\|\boldsymbol{E}^{n}\right\|_{1}^{2}+\left\|\boldsymbol{J}^{n+\frac{1}{2}}\right\|_{1}^{2}+\left\|H_{z}^{n+\frac{1}{2}}\right\|_{1}^{2}+\left\|K_{z}^{n+1}\right\|_{1}^{2} \leq C\left\{\left\|\boldsymbol{E}^{0}\right\|_{1}^{2}+\left\|\boldsymbol{J}^{\frac{1}{2}}\right\|_{1}^{2}+\left\|H_{z}^{\frac{1}{2}}\right\|_{1}^{2}+\left\|K_{z}^{1}\right\|_{1}^{2}\right\},
$$


where, for $n \geq 0$

$$
\begin{array}{ll}
\left\|E^{n}\right\|_{1}^{2}=\left\|E_{x}^{n}\right\|_{E_{x}}^{2}+\left\|E_{y}^{n}\right\|_{E_{y}}^{2}+\left|\left(E_{x}^{n}, E_{y}^{n}\right)\right|_{1}^{2}, & \left\|J^{n+\frac{1}{2}}\right\|_{1}^{2}=\left\|J_{x}^{n+\frac{1}{2}}\right\|_{J_{x}}^{2}+\left\|J_{y}^{n+\frac{1}{2}}\right\|_{J_{y}}^{2}+\left|\left(J_{x}^{n+\frac{1}{2}}, J_{y}^{n+\frac{1}{2}}\right)\right|_{1}^{2}, \\
\left\|H_{z}^{n+\frac{1}{2}}\right\|_{1}^{2}=\left\|H_{z}^{n+\frac{1}{2}}\right\|_{H_{z}}^{2}+\left|H_{z}^{n+\frac{1}{2}}\right|_{1}^{2}, \quad\left\|K_{z}^{n+1}\right\|_{1}^{2}=\left\|K_{z}^{n+1}\right\|_{H_{z}}^{2}+\left|K_{z}^{n+1}\right|_{1}^{2} .
\end{array}
$$

This indicates that the fully discrete Yee scheme is conditionally stable in the discrete $H^{1}$ norm when the time step size constraint

$$
\Delta t<\min \left\{\frac{\sqrt{\mu_{0} \epsilon_{0}}}{2} \sqrt{\frac{h_{\min }^{2} k_{\min }^{2}}{h_{\min }^{2}+k_{\min }^{2}}}, \frac{\sqrt{2}}{\sqrt{3} \omega_{p e}}, \frac{1}{\omega_{p m}}\right\}
$$

is satisfied.

\section{Numerical results and discussion}

In this section, some numerical experiments using the Yee scheme on non-uniform meshes have been carried out. We use Examples 4.1 and 4.2 to demonstrate the new energy stability, and investigate the convergence rates in discrete $L^{2}$ and $H^{1}$ norms on non-uniform rectangular and cubic meshes, respectively. In Example 4.3, we will numerically simulate the electromagnetic wave propagation in the metamaterial to show the backward wave propagation phenomenon. All our tests were carried out using MATLAB 2017b running on Dell Inspiron 7420 laptop with 12GB of RAM and 2.60GHz CPU.

In $2 \mathrm{D}$ case, denote the values of the exact solution of metamaterial Maxwell's equations (2.1)-(2.8) at the staggered points by $E_{x}\left(t^{n}\right)_{i+\frac{1}{2}, j}, E_{y}\left(t^{n}\right)_{i, j+\frac{1}{2}}, H_{z}\left(t^{n+\frac{1}{2}}\right)_{i+\frac{1}{2}, j+\frac{1}{2}}$, $J_{x}\left(t^{n+\frac{1}{2}}\right)_{i+\frac{1}{2}, j} J_{y}\left(t^{n+\frac{1}{2}}\right)_{i, j+\frac{1}{2}}$ and $K_{z}\left(t^{n+1}\right)_{i+\frac{1}{2}, j+\frac{1}{2}}$ define the error of Yee scheme as follows

$$
\begin{array}{ll}
\mathcal{E}_{x_{i+\frac{1}{2}, j}^{n}}^{n}=E_{x}\left(t^{n}\right)_{i+\frac{1}{2}, j}-E_{x_{i+\frac{1}{2}, j^{\prime}}{ }^{\prime}} & \mathcal{E}_{y_{i, j+\frac{1}{2}}^{n}}^{n}=E_{y}\left(t^{n}\right)_{i, j+\frac{1}{2}}-E_{y_{i, j+\frac{1}{2}}{ }^{n}} \\
\mathcal{J}_{x_{i+\frac{1}{2}, j}^{n+\frac{1}{2}}}=J_{x}\left(t^{n+\frac{1}{2}}\right)_{i+\frac{1}{2}, j}-J_{x_{i+\frac{1}{2}, j^{\prime}}^{n+\frac{1}{2}}} & \mathcal{J}_{y_{i, j+\frac{1}{2}}^{n+\frac{1}{2}}}=J_{y}\left(t^{n+\frac{1}{2}}\right)_{i, j+\frac{1}{2}}-J_{y_{i, j+\frac{1}{2}}^{n+\frac{1}{2}}} \\
\mathcal{H}_{z_{i+\frac{1}{2}, j+\frac{1}{2}}^{n+\frac{1}{2}}}=H_{z}\left(t^{n+\frac{1}{2}}\right)_{i+\frac{1}{2}, j+\frac{1}{2}}-H_{z_{i+\frac{1}{2}, j+\frac{1}{2}}^{n+\frac{1}{2}}}, & \mathcal{K}_{z_{i+\frac{1}{2}, j+\frac{1}{2}}^{n+1}}=K_{z}\left(t^{n+1}\right)_{i+\frac{1}{2}, j+\frac{1}{2}}-K_{z_{i+\frac{1}{2}, j+\frac{1}{2}}^{n+1}}
\end{array}
$$

Denote $E_{H 1 s 2 D}^{n}$ and $E_{L 22 D}^{n}$ as the energy in the $H^{1}$ semi-norm and in the $L^{2}$ norm, respectively.

$$
\begin{aligned}
E_{H 1 s 2 D}^{n}=\| & \delta_{x} E_{x}^{n}\left\|_{\delta_{x} E_{x}}^{2}+\right\| \delta_{x} E_{y}^{n}\left\|_{\delta_{x} E_{y}}^{2}+\right\| \delta_{y} E_{x}^{n}\left\|_{\delta_{y} E_{x}}^{2}+\right\| \delta_{y} E_{y}^{n} \|_{\delta_{y} E_{y}}^{2} \\
& +\left\|\delta_{x} J_{x}^{n+\frac{1}{2}}\right\|_{\delta_{x} J_{x}}^{2}+\left\|\delta_{x} J_{y}^{n+\frac{1}{2}}\right\|_{\delta_{x} J_{y}}^{2}+\left\|\delta_{y} J_{x}^{n+\frac{1}{2}}\right\|_{\delta_{y} J_{x}}^{2}+\left\|\delta_{y} J_{y}^{n+\frac{1}{2}}\right\|_{\delta_{y} J_{y}}^{2} \\
& +\left\|\delta_{x} H_{z}^{n+\frac{1}{2}}\right\|_{\delta_{x} H_{z}}^{2}+\left\|\delta_{y} H_{z}^{n+\frac{1}{2}}\right\|_{\delta_{y} H_{z}}^{2}+\left\|\delta_{x} K_{z}^{n+1}\right\|_{\delta_{x} K_{z}}^{2}+\left\|\delta_{y} K_{z}^{n+1}\right\|_{\delta_{y} K_{z}}^{2}
\end{aligned}
$$




$$
E_{L 22 D}^{n}=\left\|E_{x}^{n}\right\|_{E_{x}}^{2}+\left\|E_{y}^{n}\right\|_{E_{y}}^{2}+\left\|J_{x}^{n+\frac{1}{2}}\right\|_{J_{x}}^{2}+\left\|J_{y}^{n+\frac{1}{2}}\right\|_{J_{y}}^{2}+\left\|H_{z}^{n+\frac{1}{2}}\right\|_{H_{z}}^{2}+\left\|K_{z}^{n+1}\right\|_{K_{z}}^{2} .
$$

Let ErrH1s2D and ErrL22D be the absolute errors in the $H^{1}$ semi-norm and in the $L^{2}$ norm, respectively.

$$
\begin{aligned}
\operatorname{ErrH1s2D}= & \left(\left\|\delta_{x} \mathcal{E}_{x}^{n}\right\|_{\delta_{x} E_{x}}^{2}+\left\|\delta_{x} \mathcal{E}_{y}^{n}\right\|_{\delta_{x} E_{y}}^{2}+\left\|\delta_{y} \mathcal{E}_{x}^{n}\right\|_{\delta_{y} E_{x}}^{2}+\left\|\delta_{y} \mathcal{E}_{y}^{n}\right\|_{\delta_{y} E_{y}}^{2}\right. \\
& +\left\|\delta_{x} \mathcal{J}_{x}^{n+\frac{1}{2}}\right\|_{\delta_{x} J_{x}}^{2}+\left\|\delta_{x} \mathcal{J}_{y}^{n+\frac{1}{2}}\right\|_{\delta_{x} J_{y}}^{2}+\left\|\delta_{y} \mathcal{J}_{x}^{n+\frac{1}{2}}\right\|_{\delta_{y} J_{x}}^{2}+\left\|\delta_{y} \mathcal{J}_{y}^{n+\frac{1}{2}}\right\|_{\delta_{y} J_{y}}^{2} \\
& \left.+\left\|\delta_{x} \mathcal{H}_{z}^{n+\frac{1}{2}}\right\|_{\delta_{x} H_{z}}^{2}+\left\|\delta_{y} \mathcal{H}_{z}^{n+\frac{1}{2}}\right\|_{\delta_{y} H_{z}}^{2}+\left\|\delta_{x} \mathcal{K}_{z}^{n+1}\right\|_{\delta_{x} K_{z}}^{2}+\left\|\delta_{y} \mathcal{K}_{z}^{n+1}\right\|_{\delta_{y} K_{z}}^{2}\right)^{\frac{1}{2}}, \\
\operatorname{ErrL22D}=( & \left.\left\|\mathcal{E}_{x}^{n}\right\|_{E_{x}}^{2}+\left\|\mathcal{E}_{y}^{n}\right\|_{E_{y}}^{2}+\left\|\mathcal{J}_{x}^{n+\frac{1}{2}}\right\|_{J_{x}}^{2}+\left\|\mathcal{J}_{y}^{n+\frac{1}{2}}\right\|_{J_{y}}^{2}+\left\|\mathcal{H}_{z}^{n+\frac{1}{2}}\right\|_{H_{z}}^{2}+\left\|\mathcal{K}_{z}^{n+1}\right\|_{K_{z}}^{2}\right)^{\frac{1}{2}} .
\end{aligned}
$$

Example 4.1. We choose a rectangular physical domain $\Omega=(0,1) \times(0,1)$ and $\epsilon_{0}=\mu_{0}=1$, $\Gamma_{m}=\Gamma_{e}=\omega_{p m}=\omega_{p e}=\pi$. The spatial partition is $[0: h: 0.5-h, 0.5: h / 2: 1] \times[0: k: 0.5-k, 0.5$ : $k / 2: 1]$, which is the same as in [14]. The exact solution of this $2 \mathrm{D}$ problem is given by:

$$
\left\{\begin{array}{l}
\mathbf{E}=\mathbf{W} e^{-\pi t}, \quad H_{z}=\cos (\pi x) \cos (\pi y) e^{-\pi t}, \\
\mathbf{J}=\mathbf{W} e^{-\pi t} \pi^{2} t, \quad K_{z}=\cos (\pi x) \cos (\pi y) e^{-\pi t} \pi^{2} t \\
\mathbf{g}=\mathbf{W} e^{-\pi t} \pi^{2} t, \\
f=\cos (\pi x) \cos (\pi y) e^{-\pi t}\left(-3 \pi+\pi^{2} t\right)
\end{array}\right.
$$

where $\mathbf{g}=\left(g_{x}, g_{y}\right)^{T}, f$ are the right-hand side source term of Eqs. (2.1)-(2.3), respectively, $\mathbf{W}=(\cos (\pi x) \sin (\pi y),-\sin (\pi x) \cos (\pi y))^{T}$.

In order to verify the Theorems 3.1 and 3.2, we consider (4.1) without source term. We take the mesh size $h=k=1 / 64$, then $h_{\min }=k_{\min }=1 / 128$, the time step size constraint as following

$$
\Delta t<\min \left\{\frac{1}{2} \sqrt{\frac{h_{\min }^{2} k_{\min }^{2}}{h_{\min }^{2}+k_{\min }^{2}}}, \frac{\sqrt{2}}{\sqrt{3} \pi}, \frac{1}{\pi}\right\}=2.8 \times 10^{-3} .
$$

Thus, we can choose $\Delta t=10^{-3}, C=3$ in Theorems 3.1 and 3.2, then run total 10000 time steps. The energy stability results are presented in Fig. 1. From Fig. 1, we can clearly the energy in $H^{1}$ sei-norm and $L^{2}$ norm at any time are bounded by three times the initial energy.

Inspired by the result of [14], the covergence result in the $L^{2}$ norm of Yee scheme is superconvergence on non-uniform rectangular meshes. Here, we make a thorough inquiry about he convergence result in the $H^{1}$ semi-norm of Yee sheme. We take the mesh step sizes $h=k$ varying from $1 / 4$ to $1 / 256$, then, choose the fixed time step size $\Delta t=$ 


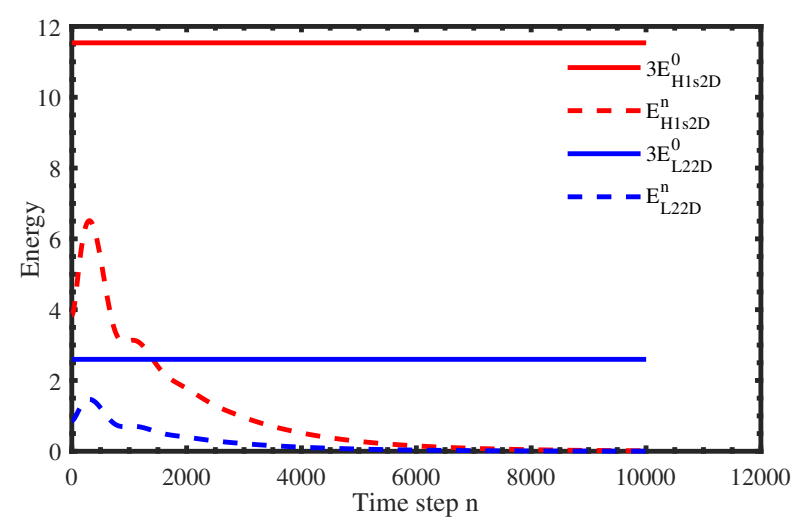

Figure 1: Time evolution of energy in $H^{1}$ semi-norm and $L^{2}$ norm in Example 4.1.

Table 1: Errors and convergence rates of the Yee scheme on non-uniform rectangular grids.

\begin{tabular}{||ccccc||}
\hline$h$ & ErrH1s2D & Rate & ErrL22D & Rate \\
\hline $1 / 4$ & $4.1889 \mathrm{E}-02$ & - & $7.8334 \mathrm{E}-03$ & - \\
$1 / 8$ & $1.1201 \mathrm{E}-02$ & 1.9030 & $1.9518 \mathrm{E}-03$ & 2.0048 \\
$1 / 16$ & $2.8803 \mathrm{E}-03$ & 1.9593 & $4.8781 \mathrm{E}-04$ & 2.0004 \\
$1 / 32$ & $7.2943 \mathrm{E}-04$ & 1.9814 & $1.2195 \mathrm{E}-04$ & 2.0001 \\
$1 / 64$ & $1.8348 \mathrm{E}-04$ & 1.9911 & $3.0485 \mathrm{E}-05$ & 2.0001 \\
$1 / 128$ & $4.6006 \mathrm{E}-05$ & 1.9958 & $7.6204 \mathrm{E}-06$ & 2.0002 \\
$1 / 256$ & $1.1515 \mathrm{E}-05$ & 1.9983 & $1.9042 \mathrm{E}-06$ & 2.0007 \\
\hline
\end{tabular}

$10^{-4}$, run total 10000 time steps to calculate the absolute errors, ErrH1s2D and ErrL22D, respectively. The numerical results are presented in Table 1. From Table 1, we can clearly see the Yee scheme on non-uniform rectangular grids is stable and the convergence rate in space is approximate second order. This shows that the Yee scheme on non-uniform rectangular grids is superconvergence in the discrete $H^{1}$ norm.

Similar to the definition of the energy and the absolute errors in 2D case, we can easily define $E_{H 1 s 3 D}^{n}, E_{L 23 D}^{n}, E r r H 1 s 3 D$ and ErrL23D, in the 3D case

$$
\begin{aligned}
& E_{H 1 s 3 D}^{n}=\left\|\delta E^{n}\right\|_{\delta E}^{2}+\left\|\delta H^{n+\frac{1}{2}}\right\|_{\delta H}^{2}+\left\|\delta J^{n+\frac{1}{2}}\right\|_{\delta J}^{2}+\left\|\delta K^{n+1}\right\|_{\delta K^{\prime}}^{2} \\
& E_{L 23 D}^{n}=\left\|E_{x}^{n}\right\|_{E_{x}}^{2}+\left\|E_{y}^{n}\right\|_{E_{y}}^{2}++\left\|E_{z}^{n}\right\|_{E_{z}}^{2}+\left\|J_{x}^{n+\frac{1}{2}}\right\|_{J_{x}}^{2}+\left\|J_{y}^{n+\frac{1}{2}}\right\|_{J_{y}}^{2}++\left\|J_{z}^{n+\frac{1}{2}}\right\|_{J_{z}}^{2} \\
& +\left\|H_{x}^{n+\frac{1}{2}}\right\|_{H_{x}}^{2}+\left\|H_{y}^{n+\frac{1}{2}}\right\|_{H_{y}}^{2}+\left\|H_{z}^{n+\frac{1}{2}}\right\|_{H_{z}}^{2}+\left\|K_{x}^{n+1}\right\|_{K_{x}}^{2}+\left\|K_{y}^{n+1}\right\|_{K_{y}}^{2}+\left\|K_{z}^{n+1}\right\|_{K_{z}}^{2}, \\
& \operatorname{ErrH1s3D}=\left(\left\|\delta \mathcal{E}^{n}\right\|_{\delta E}^{2}+\left\|\delta \mathcal{H}^{n+\frac{1}{2}}\right\|_{\delta H}^{2}+\left\|\delta \mathcal{J}^{n+\frac{1}{2}}\right\|_{\delta J}^{2}+\left\|\delta \mathcal{K}^{n+1}\right\|_{\delta K}^{2}\right)^{\frac{1}{2}} \text {, }
\end{aligned}
$$




$$
\begin{aligned}
\operatorname{ErrL23D}= & \left(\left\|\mathcal{E}_{x}^{n}\right\|_{E_{x}}^{2}+\left\|\mathcal{E}_{y}^{n}\right\|_{E_{y}}^{2}++\left\|\mathcal{E}_{z}^{n}\right\|_{E_{z}}^{2}+\left\|\mathcal{J}_{x}^{n+\frac{1}{2}}\right\|_{J_{x}}^{2}+\left\|\mathcal{J}_{y}^{n+\frac{1}{2}}\right\|_{J_{y}}^{2}++\left\|\mathcal{J}_{z}^{n+\frac{1}{2}}\right\|_{J_{z}}^{2}\right. \\
& \left.+\left\|\mathcal{H}_{x}^{n+\frac{1}{2}}\right\|_{H_{x}}^{2}+\left\|\mathcal{H}_{y}^{n+\frac{1}{2}}\right\|_{H_{y}}^{2}+\left\|\mathcal{H}_{z}^{n+\frac{1}{2}}\right\|_{H_{z}}^{2}+\left\|\mathcal{K}_{x}^{n+1}\right\|_{K_{x}}^{2}+\left\|\mathcal{K}_{y}^{n+1}\right\|_{K_{y}}^{2}+\left\|\mathcal{K}_{z}^{n+1}\right\|_{K_{z}}^{2}\right)^{\frac{1}{2}},
\end{aligned}
$$

where

$$
\begin{aligned}
\left\|\delta \mathcal{V}^{n}\right\|_{\delta \mathcal{V}}^{2}=\| & \delta_{x} \mathcal{V}_{x}^{n}\left\|_{\delta_{x} V_{x}}^{2}+\right\| \delta_{x} \mathcal{V}_{y}^{n}\left\|_{\delta_{x} V_{y}}^{2}+\right\| \delta_{x} \mathcal{V}_{z}^{n}\left\|_{\delta_{x} V_{z}}^{2}+\right\| \delta_{y} \mathcal{V}_{x}^{n} \|_{\delta_{y} V_{x}}^{2} \\
& +\left\|\delta_{y} \mathcal{V}_{y}^{n}\right\|_{\delta_{y} V_{y}}^{2}+\left\|\delta_{y} \mathcal{V}_{z}^{n}\right\|_{\delta_{y} V_{z}}^{2}+\left\|\delta_{z} \mathcal{V}_{x}^{n}\right\|_{\delta_{z} V_{x}}^{2}+\left\|\delta_{z} \mathcal{V}_{y}^{n}\right\|_{\delta_{z} V_{y}}^{2}+\left\|\delta_{z} \mathcal{V}_{z}^{n}\right\|_{\delta_{z} V_{z}}^{2}
\end{aligned}
$$

Example 4.2. In this example, we choose the physical domain is the unit cube $\Omega=[0,1]^{3}$ and all physical parameters being one (i.e., $\epsilon_{0}=\mu_{0}=\Gamma_{m}=\Gamma_{e}=\omega_{p m}=\omega_{p e}=1$ ). The spatial partition is $[0: h: 0.5-h, 0.5: h / 2: 1] \times[0: k: 0.5-k, 0.5: k / 2: 1] \times[0: l: 0.5-l, 0.5: l / 2: 1]$, which is the similar with Example 4.1. The initial condition and the right side of the equation are computed according to the analytic solution [6] given as below.

$$
\begin{aligned}
& \mathbf{E}=\mathbf{U} e^{-t} \cos t, \quad \mathbf{J}=\mathbf{U} e^{-t} \sin t, \\
& \mathbf{H}=\mathbf{V} e^{-t} \cos t, \quad \mathbf{K}=\mathbf{V} e^{-t} \sin t,
\end{aligned}
$$

where

$$
\mathbf{U}=\left(\begin{array}{c}
A \cos \pi x \sin \pi y \sin \pi z \\
B \sin \pi x \cos \pi y \sin \pi z \\
C \sin \pi x \sin \pi y \cos \pi z
\end{array}\right), \quad \mathbf{V}=\left(\begin{array}{c}
\pi(C-B) \sin \pi x \cos \pi y \cos \pi z \\
\pi(A-C) \cos \pi x \sin \pi y \cos \pi z \\
\pi(B-A) \cos \pi x \cos \pi y \sin \pi z
\end{array}\right) .
$$

The source term at the right hand side of the equation can be derived as follow

$$
\mathbf{f}=\epsilon_{0} \frac{\partial \mathbf{E}}{\partial t}-\nabla \times \mathbf{H}+\mathbf{J}=\left(\begin{array}{c}
\left(-A-3 A \pi^{2}\right) \cos \pi x \sin \pi y \sin \pi z \\
\left(-B-3 B \pi^{2}\right) \sin \pi x \cos \pi y \sin \pi z \\
\left(-C-3 C \pi^{2}\right) \sin \pi x \sin \pi y \cos \pi z
\end{array}\right) e^{-t} \cos t,
$$

where the constants $A=1, B=1 / 3$ and $C=-4 / 3$.

In this example, we explore the energy stability and convergence results of Yee scheme on nonuniform rectangular meshes in three dimensional. For the energy stability test, we consider (4.3) without source term. We take the mesh size $h=k=l=1 / 32$, then, we can choose proper parameters $\Delta t=10^{-3}, C=1$, then run total 10000 time steps. The energy stability results are presented in Fig. 2. In Fig. 2, the energy curves show that energy in $H^{1}$ sei-norm and $L^{2}$ norm for all time steps are bounded by the initial energy.

In this example, we take a fixed time step size $\Delta t=10^{-4}$, the mesh step sizes $h=k=l$ varying from $1 / 4$ to $1 / 64$ and then runs total 10000 time steps to calculate the absolute errors, ErrH1s3D and ErrL23D, in the $H^{1}$ semi-norm and $L^{2}$ norm, respectively. The numerical results are presented in Table 2. From Table 2, we can clearly see the Yee scheme on non-uniform cubic grids is superconvergence in the discrete $H^{1}$ norm. 


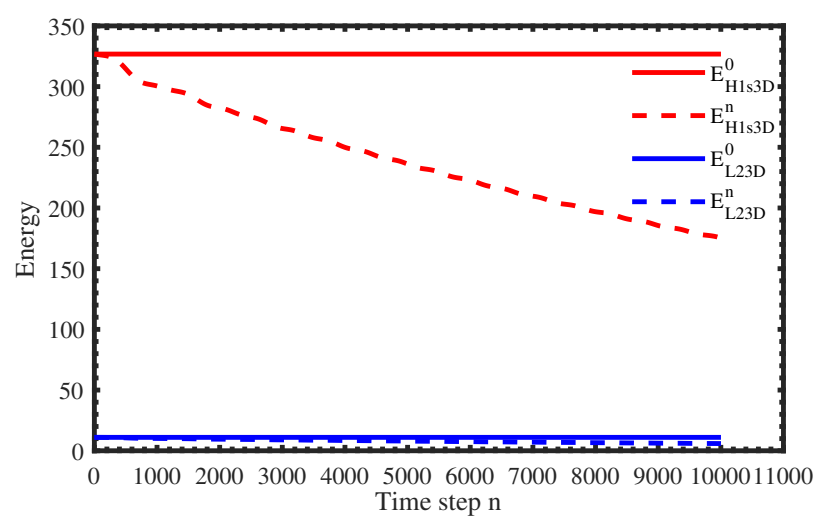

Figure 2: Time evolution of energy in $H^{1}$ semi-norm and $L^{2}$ norm in Example 4.2.

Table 2: Errors and convergence rates of the Yee scheme on non-uniform cubic grids.

\begin{tabular}{||ccccc||}
\hline$h$ & ErrH1s3D & Rate & ErrL23D & Rate \\
\hline $1 / 4$ & $2.2247 \mathrm{E}-01$ & - & $3.9970 \mathrm{E}-02$ & - \\
$1 / 8$ & $5.7572 \mathrm{E}-02$ & 1.9502 & $1.0052 \mathrm{E}-02$ & 1.9914 \\
$1 / 16$ & $1.4616 \mathrm{E}-02$ & 1.9778 & $2.5170 \mathrm{E}-03$ & 1.9978 \\
$1 / 32$ & $3.6810 \mathrm{E}-03$ & 1.9894 & $6.2948 \mathrm{E}-04$ & 1.9995 \\
$1 / 64$ & $9.2385 \mathrm{E}-04$ & 1.9944 & $1.5738 \mathrm{E}-04$ & 1.9999 \\
$1 / 128$ & $2.3135 \mathrm{E}-04$ & 1.9976 & $3.9343 \mathrm{E}-05$ & 2.0001 \\
\hline
\end{tabular}

Example 4.3. In this example, we use the Yee scheme to simulate the classic example of electromagnetic wave propagations on a non-uniform rectangular grids in metamaterials originally introduced by Ziolkowski [33]. The metamaterial slab of size $[0.024,0.054] \mathrm{m} \times$ $[0.002,0.062] \mathrm{m}$ occupies in subdomain $[0,0.07] \mathrm{m} \times[0,0.064] \mathrm{m}$ and the other domain is filled with vacuum with $\epsilon_{0}$ and $\mu_{0}$. In addition, the perfectly matched layers (PML) around the physical domain. The velocity in vacuum is $c_{0}=1 / \sqrt{\epsilon_{0} \mu_{0}}=3.0 \times 10^{8} \mathrm{~m} / \mathrm{s}$, and the frequency is chosen as $f_{0}=3 \times 10^{10} \mathrm{~Hz}$. The $H_{z}$ filed are excited with a line source located at $x=0.004 \mathrm{~m}$ and $y \in[0.025,0.035] \mathrm{m}$. The input signal in space as $e^{-(x-0.03)^{2} /(0.01)^{2}}$ and in time:

$$
f(t)= \begin{cases}g_{\mathrm{on}} \sin \left(\omega_{0} t\right) & \text { for } 0 \leq t<m T_{p} \\ \sin \left(\omega_{0} t\right) & \text { for } \quad m T_{p} \leq t<(m+n) T_{p} \\ g_{\text {off }} \sin \left(\omega_{0} t\right) & \text { for } \quad(m+n) T_{p} \leq t<(2 m+n) T_{p} \\ 0 & \text { for } \quad(2 m+n) \leq t\end{cases}
$$

where $T_{p}=1 / f_{0}$, and $g_{\text {on }}(t)=10 x_{\text {on }}^{3}-15 x_{\text {on }}^{4}+6 x_{\text {on }}^{5}, g_{\text {off }}(t)=1-\left[10 x_{\text {off }}^{3}-15 x_{\text {off }}^{4}+6 x_{\text {off }}^{5}\right]$ with $x_{\text {on }}=t / m T_{p}$ and $x_{\text {off }}=\left(t-(m+n) T_{p}\right) / m T_{p}$.

In this simulation, we take $m=2, n=12, \omega_{0}=2 \pi f_{0}, h=10^{-4} \mathrm{~m}$, the metamaterial 


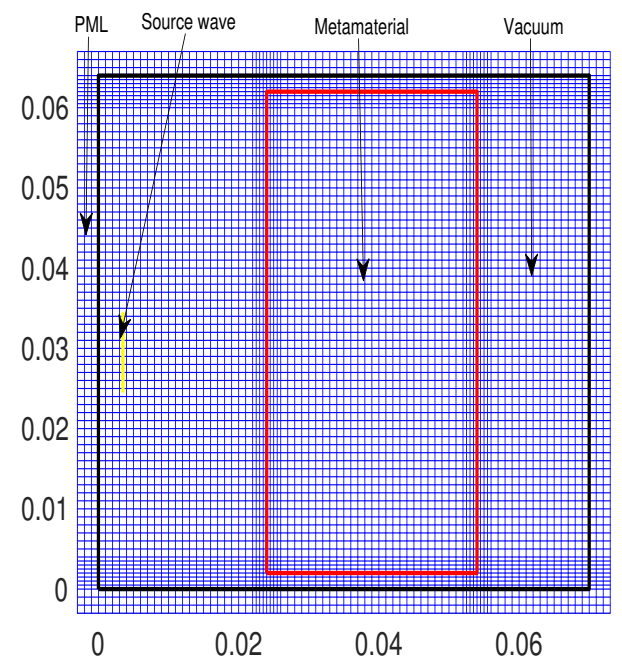

Figure 3: The non-uniform mesh sketch.

parameters as following:

$$
\begin{array}{ll}
\Gamma_{e}=\Gamma_{m}=10^{8} \mathrm{~s}^{-1}, & \omega_{p e}=\omega_{p m}=2 \pi \sqrt{2} \cdot 3 \times 10^{9} \mathrm{~s}^{-1}, \\
\epsilon_{0}=8.85 \times 10^{-12} \mathrm{C}^{2} \mathrm{~N}^{-1} \mathrm{~m}^{-2}, & \mu_{0}=4 \pi \times 10^{-7} \mathrm{H},
\end{array}
$$

the time step size $\Delta t=10^{-13} \mathrm{~s}=0.1 \mathrm{ps}$ (picosecond), run 5000 time steps.

The initial spatial partition in Fig. 3, then the non-uniform mesh uniformly refined by dividing each edge into two equal parts. The calculated $\left|H_{z}\right|$ fields and $\mathbf{E}$ fields at various times are presented in Fig. 4 . The simulation clearly shows the special phenomena (backward wave propagation) in metamaterials.

\section{Conclusions}

In this paper, several new energy identities of Maxwell's equations in metamaterials with the PEC boundary condition have been derived. These identities give us new energy methods in studying Yee scheme on non-uniform meshes. It was proved that the Yee scheme of metamaterial Maxwell's equations with the PEC boundary condition on nonuniform meshes is conditionally stable in the discrete $L^{2}$ and $H^{1}$ norms. Numerical experiments confirm the analysis on stability of Yee scheme. Moreover, we find that the Yee scheme has superconvergence in discrete $H^{1}$ and $L^{2}$ norms on non-uniform rectangular and cubic meshes. In the future, we will give the convergence analysis of the superconvergence in discrete $H^{1}$ norm. 

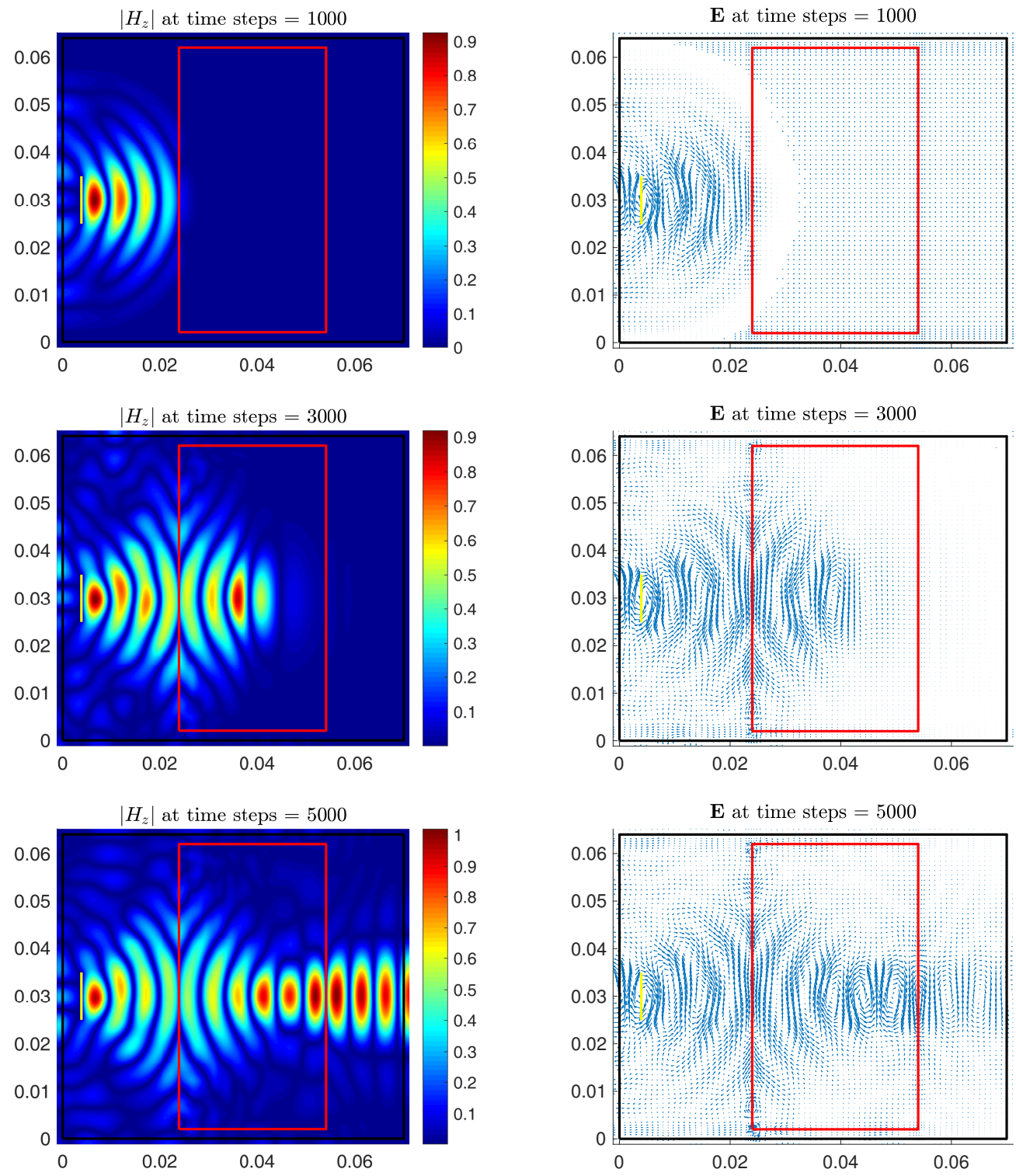

Figure 4: $\left|H_{z}\right|$ fields (left) and E fields (right) at various time steps.

\section{Acknowledgements}

The authors would like to thank the referees for their comments which have improved the paper greatly. This work is supported by the National Natural Science Foundation of China Grant No. 11671233 and the Shandong Provincial Science and Technology Devel- 
opment Program Grant No. 2018GGX101036.

\section{References}

[1] Alexander Benz, S. Campione, S. Liu, I. Montano, J. F. Klem, A. Allerman, J. R. Wendt, M. B. Sinclair, F. CAPOlino, AND I. BRENER, Strong coupling in the subwavelength limit using metamaterial nanocavities, Nat. Commun., 4(1) (2013), pp. 1-8.

[2] Wenbin CHEN, XINGJIE LI, AND DONG LIANG, Energy-conserved splitting FDTD methods for Maxwell's equations, Numer. Math., 108(3) (2008), pp. 445-485.

[3] Liping GaO, Minmin CaO, Rengang Shi, AND Hui GuO, Energy conservation and super convergence analysis of the EC-S-FDTD schemes for Maxwell equations with periodic boundaries, Numer. Math. Partial Differential Equations, 35(4) (2019).

[4] LiPING GAO AND BO ZHANG, Stability and superconvergence analysis of the FDTD scheme for the 2D Maxwell equations in a lossy medium, Sci. China. Math., 54(12) (2011), pp. 2693-2712.

[5] Liping GAO, BO ZHANG, AND DONG LIANG, Splitting finite difference methods on staggered grids for the three-dimensional time-dependent Maxwell equations, Commun. Comput. Phys., $4(2)$ (2008), pp. 405-432.

[6] YUNQING HuANG, Jichun Li, Wei YANG, AND ShuYu Sun, Superconvergence of mixed finite element approximations to 3-D Maxwell's equations in metamaterials, J. Comput. Phys., 230(22) (2011), pp. 8275-8289.

[7] LinghUA KONG, JiAlin HONG, AND JingJing ZHANG, Splitting multisymplectic integrators for Maxwell's equations, J. Comput. Phys., 229(11) (2010), pp. 4259-4278.

[8] Ulf LeONHARDT, Optical conformal mapping, Science, 312(5781) (2006), pp. 1777-1780.

[9] JICHUN LI, Error analysis of mixed finite element methods for wave propagation in double negative metamaterials, J. Comput. Appl. Math., 209(1) (2007), pp. 81-96.

[10] Jichun Li, MENG CHEN, AND Min CHen, Developing and analyzing fourth-order difference methods for the metamaterial maxwell's equations, Adv. Comput. Math., 45(1) (2019), pp. 213241.

[11] JiChun Li AND JAn S. HesthaVen, Analysis and application of the nodal discontinuous Galerkin method for wave propagation in metamaterials, J. Comput. Phys., 258 (2014), pp. 915930.

[12] Jichun Li And YunQIng Huang, Time-Domain Finite Element Methods for Maxwell's Equations in Metamaterials, volume 43, Springer Science, Business Media, 2012.

[13] Jichun Li, CengKe ShI, AND ChI-WANG SHu, Optimal non-dissipative discontinuous Galerkin methods for Maxwell's equations in Drude metamaterials, Comput. Math. Appl., 73(8) (2017), pp. 1760-1780.

[14] JiCHUN Li AND SIDNEy SHIELDS, Superconvergence analysis of Yee scheme for metamaterial Maxwell's equations on non-uniform rectangular meshes, Numer. Math., 134(4) (2016), pp. 741781.

[15] WANSHAN Li AND DONG LiAng, Symmetric energy-conserved S-FDTD scheme for twodimensional Maxwell's equations in negative index metamaterials, J. Sci. Comput., 69(2) (2016), pp. 696-735.

[16] WANShan Li, DONG Liang, AND YANPING Lin, A new energy-conserved S-FDTD scheme for maxwell's equations in metamaterials, Int. J. Numer. Anal. Model., 10(4) (2013).

[17] WANSHAN LI, DONG LIANG, AND YANPING LIN, Global energy-tracking identities and global energy-tracking splitting FDTD schemes for the Drude models of Maxwell's equations in three- 
dimensional metamaterials, Numer. Math. Partial Differential Equations, 33(3) (2017), pp. 763785.

[18] Liping, GaO, XingJie, Li, Wenbin, And Wenbin Chen, New energy identities and super convergence analysis of the energy conserved splitting FDTD methods for 3D Maxwell's equations, Math. Method. Appl. Sci., (2013).

[19] Peter MONK AND ENDRE SÜLI, A convergence analysis of Yee's scheme on nonuniform grids, SIAM J. Numer. Anal., 31(2) (1994), pp. 393-412.

[20] T. NAMIKI, A new FDTD algorithm based on alternating-direction implicit method, IEEE Trans. Microw. Theory Tech., 47(10) (1999), pp. 2003-2007.

[21] ROB F. REMIS, Stability of FDTD on nonuniform grids for Maxwell's equations in lossless media, J. Comput. Phys., 218(2) (2006), pp. 594-606.

[22] David Schurig, Jack J. Mock, B. J. Justice, Steven A. Cummer, John B. Pendry, ANTHONY F. STARR, AND DAVID R. SMITH, Metamaterial electromagnetic cloak at microwave frequencies, Science, 314(5801) (2006), pp. 977-980.

[23] Richard A. Shelby, David R. Smith, AND SEldon Schultz, Experimental verification of a negative index of refraction, Science, 292(5514) (2001), pp. 77-79.

[24] David R. Smith, Willie J. Padilla, D. C. Vier, SYrus C. Nemat-Nasser, and Seldon SCHULTZ, Composite medium with simultaneously negative permeability and permittivity, Phys. Rev. Lett., 84(18) (2000), 4184.

[25] Mankei Tsang And Demetri Psaltis, Magnifying perfect lens and superlens design by coordinate transformation, Phys. Rev. B, 77(3) (2008), 035122.

[26] ViKTOR G. VeSElago, The electrodynamics of substances with simultaneously negative values of and $\mu$, Physics-Uspekhi, 10(4) (1968), pp. 509-514.

[27] XIANG WANG, JiCHUN Li, AND ZHIWEI FANG, Development and analysis of Crank-Nicolson scheme for metamaterial Maxwell's equations on nonuniform rectangular grids, Numer. Math. Partial Differential Equations, 34(6) (2018), pp. 2040-2059.

[28] M. C. K. Wiltshire, J. B. Pendry, AND J. V. Hajnal, Sub-wavelength imaging at radio frequency, J. Phys. Condens. Matter, 18(22) (2006), pp. L315.

[29] Ziging Xie, JiangXing Wang, Bo Wang, and Chuanmiao Chen, Solving Maxwell's equation in meta-materials by a CG-DG method, Commun. Comput. Phys., 19(5) (2016), pp. $1242-1264$.

[30] Changhui YAO AND LixiU WANG, Nonconforming finite element methods for wave propagation in metamaterials, Numer. Math. Theory. Mech., 10(1) (2017), pp. 145-166.

[31] KANE YEE, Numerical solution of initial boundary value problems involving Maxwell's equations in isotropic media, IEEE Trans. Antennas Propag., 14(3) (1966), pp. 302-307.

[32] Yunding HuAng, Meng Chen, Jichun Li AND YANPING Lin, Numerical analysis of a leapfrog ADI-FDTD method for Maxwell's equations in lossy media, Comput. Math. Appl., (2018).

[33] Richard W. ZiolKowsKI, Pulsed and CW Gaussian beam interactions with double negative metamaterial slabs, Opt. Express, 11(7) (2003), pp. 662-681. 